\title{
A CLASSIFICATION OF PURE STATES ON QUANTUM SPIN CHAINS SATISFYING THE SPLIT PROPERTY WITH ON-SITE FINITE GROUP SYMMETRIES
}

\begin{abstract}
YOSHIKO OGATA
AbSTRACT. We consider a set $\operatorname{SPG}(\mathcal{A})$ of pure split states on a quantum spin chain $\mathcal{A}$ which are invariant under the on-site action $\tau$ of a finite group $G$. For each element $\omega$ in $S P G(\mathcal{A})$ we can associate a second cohomology class $c_{\omega, R}$ of $G$. We consider a classification of $\operatorname{SPG}(\mathcal{A})$ whose criterion is given as follows: $\omega_{0}$ and $\omega_{1}$ in $S P G(\mathcal{A})$ are equivalent if there are automorphisms $\Xi_{R}$, $\Xi_{L}$ on $\mathcal{A}_{R}, \mathcal{A}_{L}$ (right and left half infinite chains) preserving the symmetry $\tau$, such that $\omega_{1}$ and $\omega_{0} \circ\left(\Xi_{L} \otimes \Xi_{R}\right)$ are quasi-equivalent. It means that we can move $\omega_{0}$ close to $\omega_{1}$ without changing the entanglement nor breaking the symmetry. We show that the second cohomology class $c_{\omega, R}$ is the complete invariant of this classification.
\end{abstract}

\section{INTRODUCTION}

It is well-known that the pure state space $P(\mathcal{A})$ of a quantum spin chain $\mathcal{A}$ (UHFalgebra, see subsection (1.1) is homogeneous under the action of the asymptotically inner automorphisms $[\mathrm{P},[\mathrm{B}, \mathrm{FKK}]$. In fact, the homogeneity is proven for much larger class, i.e., for all the separable simple $C^{*}$-algebras $[\mathrm{KOS}]$.

In this paper, we focus on the subset $S P(\mathcal{A})$ of $P(\mathcal{A})$ consisting of pure states satisfying the split property. (See Definition 1.4) One equivalent condition for a state $\omega \in P(\mathcal{A})$ to satisfy the split property is that $\omega$ is quasi-equivalent to $\left.\left.\omega\right|_{\mathcal{A}_{L}} \otimes \omega\right|_{\mathcal{A}_{R}} . \quad\left(\right.$ See Remark 1.5.) Here, $\left.\omega\right|_{\mathcal{A}_{L}},\left.\omega\right|_{\mathcal{A}_{R}}$ are restrictions of $\omega$ onto the left/right half-infinite chains. (See subsection 1.1.) Recall that two state being quasi-equivalent can be understood physically that they are "macroscopically same", because it means that one state can be represented as a local perturbation of the other and vice versa. On the other hand, a product state on $\mathcal{A}=\mathcal{A}_{L} \otimes \mathcal{A}_{R}$ has no entanglement between $\mathcal{A}_{L}$ and $\mathcal{A}_{R}$ by definition. Therefore, a state with the split property can be physically understood as a state without macroscopic entanglement between the left half and the right half of the chain. Using the result of $[\mathrm{P}], \mathrm{B}]$, FKK, , KOS, one can easily see that for any $\omega_{0}, \omega_{1} \in S P(\mathcal{A})$, there exist asymptotically inner automorphisms $\Xi_{L}, \Xi_{R}$ on $\mathcal{A}_{L}, \mathcal{A}_{R}$ such that $\left.\left.\omega_{1}\right|_{\mathcal{A}_{L}} \sim_{\text {q.e. }} \omega_{0}\right|_{\mathcal{A}_{L}} \circ \Xi_{L}$ and $\left.\left.\omega_{1}\right|_{\mathcal{A}_{R}} \sim_{\text {q.e. }} \omega_{0}\right|_{\mathcal{A}_{R}} \circ \Xi_{R}$. (Here $\sim_{\text {q.e. }}$ means quasi-equivalence.) From this and the split property of $\omega_{0}, \omega_{1}$, we see that $\omega_{1}$ and $\omega_{0} \circ\left(\Xi_{L} \otimes \Xi_{R}\right)$ are quasi-equivalent. The product of automorphisms $\Xi_{L} \otimes \Xi_{R}$ clearly does not create/destroy any entanglement between the left half and the right half of the chain. Hence any $\omega_{0} \in S P(\mathcal{A})$

Received by the editors November 11, 2019, and, in revised form, June 17, 2020.

2020 Mathematics Subject Classification. Primary 46L30.

The author was supported in part by the Grants-in-Aid for Scientific Research, JSPS. This work was supported by JSPS KAKENHI Grant Number 16 K05171 and 19K03534. 
can get "close to" any $\omega_{1} \in S P(\mathcal{A})$ without changing the entanglement. In this sense, we may regard $S P(\mathcal{A})$ to be "homogeneous".

What we would like to show in this paper is that the situation changes when symmetry comes into the game. This corresponds to the notion of symmetry protected topological phases in physics [0]. Let $S P G(\mathcal{A})$ be the set of all states in $S P(\mathcal{A})$ which are invariant under the onsite action $\tau$ of a finite group $G$. (See Definition 1.4.) We now require that the automorphisms $\Xi_{L}, \Xi_{R}$ above to preserve the symmetry i.e., $\Xi_{L} \circ \tau_{L}(g)=\tau_{L}(g) \circ \Xi_{L}$ and $\Xi_{R} \circ \tau_{R}(g)=\tau_{R}(g) \circ \Xi_{R}$ for all $g \in G$. (See (3) for the definition of $\tau_{L}$ and $\tau_{R}$.) For any $\omega_{0}, \omega_{1} \in S P G(\mathcal{A})$, can we always find such automorphisms giving $\omega_{1} \sim_{\text {q.e. }} \omega_{0} \circ\left(\Xi_{L} \otimes \Xi_{R}\right)$ ? We show that the answer is no in general. The obstacle is given by the second cohomology class of the projective representation of $G$ associated to $\omega \in S P G(\mathcal{A})$. We show that this second cohomology class is the complete invariant of this classification.

1.1. Setting. We consider the setting in this subsection throughout this paper. We use the basic notation in Appendix A freely. We start by summarizing standard setup of quantum spin chains on the infinite chain BR1, BR2. Throughout this paper, we fix some $2 \leq d \in \mathbb{N}$. We denote the algebra of $d \times d$ matrices by $\mathrm{M}_{d}$.

For each subset $\Gamma$ of $\mathbb{Z}$, we denote the set of all finite subsets in $\Gamma$ by $\mathfrak{S}_{\Gamma}$. We use the notation $\Gamma_{R}=[0, \infty) \cap \mathbb{Z}$ and $\Gamma_{L}=(-\infty,-1] \cap \mathbb{Z}$.

For each $z \in \mathbb{Z}$, let $\mathcal{A}_{\{z\}}$ be an isomorphic copy of $\mathrm{M}_{d}$, and for any finite subset $\Lambda \subset \mathbb{Z}$, we set $\mathcal{A}_{\Lambda}=\bigotimes_{z \in \Lambda} \mathcal{A}_{\{z\}}$. For finite $\Lambda$, the algebra $\mathcal{A}_{\Lambda}$ can be regarded as the set of all bounded operators acting on the Hilbert space $\bigotimes_{z \in \Lambda} \mathbb{C}^{d}$. We use this identification freely. If $\Lambda_{1} \subset \Lambda_{2}$, the algebra $\mathcal{A}_{\Lambda_{1}}$ is naturally embedded in $\mathcal{A}_{\Lambda_{2}}$ by tensoring its elements with the identity. For an infinite subset $\Gamma \subset \mathbb{Z}, \mathcal{A}_{\Gamma}$ is given as the inductive limit of the algebras $\mathcal{A}_{\Lambda}$ with $\Lambda \in \mathfrak{S}_{\Gamma}$. We call $\mathcal{A}_{\Gamma}$ the quantum spin system on $\Gamma$. In particular, we use notation $\mathcal{A}:=\mathcal{A}_{\mathbb{Z}}, \mathcal{A}_{R}:=\mathcal{A}_{\Gamma_{R}}$ and $\mathcal{A}_{L}:=\mathcal{A}_{\Gamma_{L}}$. Occasionally, we call them quantum spin chain, right infinite chain, left infinite chain, respectively. Note that each of $\mathcal{A}_{\Lambda}, \mathcal{A}_{\Gamma}$ can be regarded naturally as a subalgebra of $\mathcal{A}$. We also set $\mathcal{A}_{\text {loc }, \Gamma}=\bigcup_{\Lambda \in \mathfrak{S}_{\Gamma}} \mathcal{A}_{\Lambda}$, for any $\Gamma \subset \mathbb{Z}$.

We denote the standard basis of $\mathbb{C}^{d}$ by $\left\{e_{i}\right\}_{i=1, \ldots, d}$, and denote the standard matrix unit of $\mathrm{M}_{d}$ by $\left\{E_{i, j} \mid i, j=1, \ldots, d\right\}$. Namely, $E_{i, j}$ is a matrix such that $E_{i, j} e_{k}=\delta_{j, k} e_{i}$. For each finite $\Lambda \subset \mathbb{Z}$, we denote the tensor product $\bigotimes_{k \in \Lambda} E_{i_{k}, j_{k}}$ of $E_{i_{k}, j_{k}}$ along $k \in \Lambda$, by $E_{I, J}^{(\Lambda)}$ with $I:=\left(i_{k}\right)_{k \in \Lambda}$ and $J:=\left(j_{k}\right)_{k \in \Lambda}$. We also use the notation

$$
\mathcal{S}_{\Lambda}:=\left\{E_{I, J}^{(\Lambda)} \mid I, J \in\{1, \ldots, d\}^{\times \Lambda}\right\} .
$$

Furthermore, we set $e_{I}^{(\Lambda)}:=\bigotimes_{k \in \Lambda} e_{i_{k}} \in \bigotimes_{\Lambda} \mathbb{C}^{d}$ for $I:=\left(i_{k}\right)_{k \in \Lambda}$.

Throughout this paper we fix a finite group $G$ and its unitary representation $U$ on $\mathbb{C}^{d}$ satisfying

$$
U(g) \notin \mathbb{C I}_{\mathbb{C}^{d}}, \quad \text { if } \quad g \neq e .
$$

We denote the identity of $G$ by $e$.

Let $\Gamma \subset \mathbb{Z}$ be a non-empty subset. For each $g \in G$, there exists a unique automorphism $\tau_{\Gamma}$ on $\mathcal{A}_{\Gamma}$ such that

$$
\tau_{\Gamma}(g)(a)=\operatorname{Ad}\left(\bigotimes_{I} U(g)\right)(a), \quad a \in \mathcal{A}_{I}, \quad g \in G,
$$


for any finite subset $I$ of $\Gamma$. We call the group homomorphism $\tau_{\Gamma}: G \rightarrow$ Aut $\mathcal{A}_{\Gamma}$, the on-site action of $G$ on $\mathcal{A}_{\Gamma}$ given by $U$. In particular, when $\Gamma=\mathbb{Z}$, (resp. $\Gamma=\Gamma_{R}$, $\Gamma=\Gamma_{L}$ ), we denote $\tau_{\Gamma}$ by $\tau$ (resp. $\left.\tau_{R}, \tau_{L}\right)$. For $\Gamma \subset \mathbb{Z}$, we denote by $\mathcal{A}_{\Gamma}^{G}$ the fixed point subalgebra of $\mathcal{A}_{\Gamma}$ with respect to $\tau_{\Gamma}$. For simplicity, also use the notation $\mathcal{A}_{L}^{G}:=\mathcal{A}_{\Gamma_{L}}^{G}$ and $\mathcal{A}_{R}^{G}:=\mathcal{A}_{\Gamma_{R}}^{G}$.

1.2. Projective representations of $G$. Let $\mathbb{T}:=\{z \in \mathbb{C}|| z \mid=1\}$. A map $\sigma: G \times G \rightarrow \mathbb{T}$ is called a 2-cocycle of $G$ if

(1) $\sigma(g, h) \sigma(g h, k)=\sigma(h, k) \sigma(g, h k)$, for all $g, h, k \in G$,

(2) $\sigma(g, e)=\sigma(e, g)=1$ for all $g \in G$.

Define the product of two 2-cocycles by their point-wise product. The set of all 2 -cocycles of $G$ then becomes an abelian group. The resulting group we denote by $Z^{2}(G, \mathbb{T})$. The identity of $Z^{2}(G, \mathbb{T})$ is given by $1_{Z^{2}(G, \mathbb{T})}(g, h):=1$, for $g, h \in G$. For an arbitrary function $b: G \rightarrow \mathbb{T}$ such that $b(e)=1$,

$$
\sigma_{b}(g, h)=b(g h)^{-1} b(g) b(h), \quad g, h \in G
$$

defines a 2-cocycle. The set of all 2-cocycles of this type forms a subgroup $B^{2}(G, \mathbb{T})$ of $Z^{2}(G, \mathbb{T})$. (It is clearly normal because $Z^{2}(G, \mathbb{T})$ is abelian.) The quotient group $H^{2}(G, \mathbb{T}):=Z^{2}(G, \mathbb{T}) / B^{2}(G, \mathbb{T})$ is called the second cohomology group of $G$. For each $\sigma \in Z^{2}(G, \mathbb{T})$, we denote by $[\sigma]_{H^{2}(G, \mathbb{T})}$ the second cohomology class that $\sigma$ belongs to.

A projective unitary representation of $G$ is a triple $(\mathcal{H}, V, \sigma)$ consisting of a Hilbert space $\mathcal{H}$, a map $V: G \rightarrow \mathcal{U}(\mathcal{H})$ and a 2-cocycle $\sigma$ of $G$ such that $V(g) V(h)=$ $\sigma(g, h) V(g h)$ for all $g, h \in G$. Note that we get $V(e)=\mathbb{I}_{\mathcal{H}}$ from the latter condition. We call $\sigma$, the 2-cocycle of $G$ associated to $V$, and call $[\sigma]_{H^{2}(G, \mathbb{T})}$ the second cohomology class of $G$ associated to $V$. We occasionally say $(\mathcal{H}, V)$ is a projective unitary representation with 2-cocycle $\sigma$. The character of a finite dimensional projective unitary representation $(\mathcal{H}, V, \sigma)$ is given by $\chi_{V}(g)=\operatorname{Tr}_{\mathcal{H}} V(g)$, for $g \in G$.

We say a projective unitary representation $(\mathcal{H}, V, \sigma)$ of $G$ is irreducible if $\mathcal{H}$ and 0 are the only $V$-invariant subspaces of $\mathcal{H}$. As $G$ is a finite group, for any irreducible projective unitary representation $(\mathcal{H}, V, \sigma)$ of $G$, the Hilbert space $\mathcal{H}$ is finite dimensional. Projective unitary representations $\left(\mathcal{H}_{1}, V_{1}, \sigma_{1}\right)$ and $\left(\mathcal{H}_{2}, V_{2}, \sigma_{2}\right)$ are said to be unitarily equivalent if there is a unitary $W: \mathcal{H}_{1} \rightarrow \mathcal{H}_{2}$ such that $W V_{1}(g) W^{*}=V_{2}(g)$, with $g \in G$. Clearly if $\left(\mathcal{H}_{1}, V_{1}, \sigma_{1}\right)$ and $\left(\mathcal{H}_{2}, V_{2}, \sigma_{2}\right)$ are unitarily equivalent, the 2-cocycles $\sigma_{1}$ and $\sigma_{2}$ coincides. Schur's Lemma holds: let $\left(\mathcal{H}_{1}, V_{1}, \sigma_{1}\right)$ and $\left(\mathcal{H}_{2}, V_{2}, \sigma_{2}\right)$ be irreducible projective unitary representations of $G$, and $W: \mathcal{H}_{1} \rightarrow \mathcal{H}_{2}$ be a linear map such that $W V_{1}(g)=V_{2}(g) W$ for all $g \in G$. Then either $W=0$ or $\left(\mathcal{H}_{1}, V_{1}, \sigma_{1}\right)$ and $\left(\mathcal{H}_{2}, V_{2}, \sigma_{2}\right)$ are unitarily equivalent. The proof is the same as that of the genuine representations (see [S] Theorem II.4.2 for example.)

For $\sigma \in Z^{2}(G, \mathbb{T})$, we denote by $\mathcal{P}_{\sigma}$, the set of all unitarily equivalence classes of irreducible projective representations with 2-cocycle $\sigma$. Note that $\mathcal{P}_{1_{Z^{2}(G, \mathbb{T})}}$ is equal to $\hat{G}$, the dual of $G$ i.e. the set of equivalence classes of irreducible representations.

For each $\alpha \in \mathcal{P}_{\sigma}$, we fix a representative $\left(\mathcal{H}_{\alpha}, V_{\alpha}, \sigma\right)$. We denote the dimension of $\mathcal{H}_{\alpha}$ (which is finite) by $n_{\alpha}$ and fix an orthonormal basis $\left\{\psi_{k}^{(\alpha)}\right\}_{k=1}^{n_{\alpha}}$ of $\mathcal{H}_{\alpha}$. We introduce the matrix unit $\left\{f_{k, j}^{(\alpha)} \mid k, j=1, \ldots, n_{\alpha}\right\}$ of $\mathcal{B}\left(\mathcal{H}_{\alpha}\right)$ given by

$$
f_{k, j}^{(\alpha)} \xi=\left\langle\psi_{j}^{(\alpha)}, \xi\right\rangle \psi_{k}^{(\alpha)}, \quad \xi \in \mathcal{H}_{\alpha} . \quad k, j=1, \ldots, n_{\alpha} .
$$


We will use the following vector later, in section 4

$$
\Omega_{\alpha}:=\frac{1}{\sqrt{n_{\alpha}}} \sum_{k=1}^{n_{\alpha}} \psi_{k}^{(\alpha)} \otimes \psi_{k}^{(\alpha)} \in \mathcal{H}_{\alpha} \otimes \mathcal{H}_{\alpha} .
$$

For each $\alpha \in \mathcal{P}_{\sigma}$ and $k, j=1, \ldots, n_{\alpha}$, define a function $\left(V_{\alpha}\right)_{k, j}$ on $G$ by

$$
\left(V_{\alpha}\right)_{k, j}(g):=\left\langle\psi_{k}^{(\alpha)}, V_{\alpha}(g) \psi_{j}^{(\alpha)}\right\rangle, \quad g \in G .
$$

As in Theorem III.1.1 of [S], from Schur's Lemma, we obtain the orthogonality relation:

$$
\frac{1}{|G|} \sum_{g \in G}\left(V_{\alpha}\right)_{k, j}(g) \overline{\left(V_{\beta}\right)_{t, s}(g)}=\frac{\delta_{\alpha, \beta} \delta_{j, s} \delta_{k, t}}{n_{\alpha}}
$$

for all $\alpha, \beta \in \mathcal{P}_{\sigma}$ and $k, j, t, s=1, \ldots, n_{\alpha}$. Here $|G|$ denotes the number of elements in $G$. In particular, $\mathcal{P}_{\sigma}$ is a finite set. We freely identify $\alpha$ and $V_{\alpha}$. For example, $\alpha \otimes \beta^{\prime}, \alpha \otimes V$ should be understood as $V_{\alpha} \otimes V_{\beta^{\prime}}, V_{\alpha} \otimes V$ for $\alpha \in \mathcal{P}_{\sigma}, \beta^{\prime} \in \mathcal{P}_{\sigma^{\prime}}$, and a projective unitary representation $V$. We repeatedly use the following fact.

Lemma 1.1. For any projective unitary representation $(\mathcal{H}, V, \sigma)$, there are Hilbert spaces $\mathcal{K}_{\alpha}$ labeled by $\alpha \in \mathcal{P}_{\sigma}$ and a unitary $W: \mathcal{H} \rightarrow \bigoplus_{\alpha \in \mathcal{P}_{\sigma}} \mathcal{H}_{\alpha} \otimes \mathcal{K}_{\alpha}$ such that

$$
W V(g) W^{*}=\bigoplus_{\alpha \in \mathcal{P}_{\sigma}} V_{\alpha}(g) \otimes \mathbb{I}_{\mathcal{K}_{\alpha}}, \quad g \in G
$$

Furthermore, the commutant $V(G)^{\prime}:=\{X \in \mathcal{B}(\mathcal{H}) \mid[X, V(g)]=0\}$ of $V(G)$ is of the form

$$
V(G)^{\prime}=W^{*}\left(\bigoplus_{\alpha \in \mathcal{P}_{\sigma}} \mathbb{I}_{\mathcal{H}_{\alpha}} \otimes \mathcal{B}\left(\mathcal{K}_{\alpha}\right)\right) W
$$

Proof. For any $V$-invariant subspace of $\mathcal{H}$, its orthogonal complement is $V$-invariant as well. Therefore, from Zorn's Lemma, we may decompose $(\mathcal{H}, V, \sigma)$ as an orthogonal sum of irreducible projective unitary representations with 2-cocycle $\sigma$. This proves (9). The second statement (10) follows from the orthogonality relation (8).

Notation 1.2. When (9) holds, we say that $V$ (or $(\mathcal{H}, V, \sigma)$ ) has an irreducible decomposition given by Hilbert spaces $\left\{\mathcal{K}_{\gamma} \mid \gamma \in \mathcal{P}_{\sigma}\right\}$. We say $V$ (or $(\mathcal{H}, V, \sigma)$ ) contains all elements of $\mathcal{P}_{\sigma}$ if $\mathcal{K}_{\alpha} \neq\{0\}$ for all $\alpha \in \mathcal{P}_{\sigma}$. We say $V$ (or $(\mathcal{H}, V, \sigma)$ ) contains all elements of $\mathcal{P}_{\sigma}$ with infinite multiplicity if $\operatorname{dim} \mathcal{K}_{\alpha}=\infty$ for all $\alpha \in \mathcal{P}_{\sigma}$. We hence force omit $W$ in (9) and identify $\mathcal{H}$ and $\bigoplus_{\alpha \in \mathcal{P}_{\sigma}} \mathcal{H}_{\alpha} \otimes \mathcal{K}_{\alpha}$ freely. The Hilbert space $\mathcal{H}_{\alpha} \otimes \mathcal{K}_{\alpha}$ can be naturally regarded as a closed subspace of $\mathcal{H}$. We use this identification freely and call $\mathcal{H}_{\alpha} \otimes \mathcal{K}_{\alpha}$ the $\alpha$-component of $V($ or $(\mathcal{H}, V, \sigma))$.

Notation 1.3. Let $(\mathcal{H}, V, \sigma)$ be a projective unitary representation. Let $b: G \rightarrow \mathbb{T}$ be a map such that $b(e)=1$. Setting $\sigma_{b}$ as in (4), we obtain $\sigma \sigma_{b} \in Z^{2}(G, \mathbb{T})$. We also set $(b \cdot V)(g):=b(g) V(g)$, for $g \in G$. Then $\left(\mathcal{H}, b \cdot V, \sigma \sigma_{b}\right)$ is a projective representation. 
1.3. The split property and projective representations. Next let us introduce the split property.

Definition 1.4. Let $\omega$ be a pure state on $\mathcal{A}$. Let $\omega_{R}$ be the restriction of $\omega$ to $\mathcal{A}_{R}$, and $\left(\mathcal{H}_{\omega_{R}}, \pi_{\omega_{R}}, \Omega_{\omega_{R}}\right)$ be the GNS triple of $\omega_{R}$. We say $\omega$ satisfies the split property with respect to $\mathcal{A}_{L}$ and $\mathcal{A}_{R}$, if the von Neumann algebra $\pi_{\omega_{R}}\left(\mathcal{A}_{R}\right)^{\prime \prime}$ is a type I factor. We denote by $S P(\mathcal{A})$ the set of all pure states on $\mathcal{A}$ which satisfy the split property with respect to $\mathcal{A}_{L}$ and $\mathcal{A}_{R}$. We also denote by $S P G(\mathcal{A})$, the set of all states $\omega$ in $S P(\mathcal{A})$, which are $\tau$-invariant.

Recall that a type I factor is $*$-isomorphic to $B(\mathcal{K})$, the set of all bounded operators on a Hilbert space $\mathcal{K}$. See $[\mathrm{T}]$.

Remark 1.5. Let $\omega$ be a pure state on $\mathcal{A}$. Let $\omega_{L}$ be the restriction of $\omega$ to $\mathcal{A}_{L}$. Then $\omega$ satisfies the split property if and only if $\omega_{L} \otimes \omega_{R}$ is quasi-equivalent to $\omega$. ( See M1. In Proposition 2.2 of [M1, it is assumed that the state is translationally invariant because of the first equivalent condition (i). However, the proof for the equivalence between (ii) and (iii) does not require translation invariance.) Therefore, by the symmetric argument, if $\left(\mathcal{H}_{\omega_{L}}, \pi_{\omega_{L}}, \Omega_{\omega_{L}}\right)$ is the GNS triple of $\omega_{L}$, then the split property of $\omega$ implies that $\pi_{\omega_{L}}\left(\mathcal{A}_{L}\right)^{\prime \prime}$ is also a type I factor.

For each $\omega \in S P G(\mathcal{A})$, we may associate a second cohomology class of $G$.

Proposition 1.6. Let $\omega \in S P G(\mathcal{A})$ and $\varsigma=L, R$. Then there exists an irreducible *-representation $\rho_{\omega, \varsigma}$ of $\mathcal{A}_{\varsigma}$ on a Hilbert space $\mathcal{L}_{\omega, \varsigma}$ that is quasi-equivalent to the GNS representation of $\left.\omega\right|_{\mathcal{A}_{\varsigma}}$. For each of such irreducible *-representation $\left(\mathcal{L}_{\omega, \varsigma}, \rho_{\omega, \varsigma}\right)$, there is a projective unitary representation $u_{\omega, \varsigma}$ of $G$ on $\mathcal{L}_{\omega, \varsigma}$ such that

$$
\rho_{\omega, \varsigma} \circ \tau_{\varsigma}(g)=\operatorname{Ad}\left(u_{\omega, \varsigma}(g)\right) \circ \rho_{\omega, \varsigma},
$$

for all $g \in G$. Furthermore, if another triple $\left(\tilde{\mathcal{L}}_{\omega, \varsigma}, \tilde{\rho}_{\omega, \varsigma}, \tilde{u}_{\omega, \varsigma}\right)$ satisfies the same conditions as $\left(\mathcal{L}_{\omega, \varsigma}, \rho_{\omega, \varsigma}, u_{\omega, \varsigma}\right)$ above, then there is a unitary $W: \mathcal{L}_{\omega, \varsigma} \rightarrow \tilde{\mathcal{L}}_{\omega, \varsigma}$ and $c: G \rightarrow \mathbb{T}$ such that

$$
\begin{gathered}
(\operatorname{Ad} W) \circ \rho_{\omega, \varsigma}=\tilde{\rho}_{\omega, \varsigma}, \\
c(g) \cdot(\operatorname{Ad} W)\left(u_{\omega, \varsigma}(g)\right)=\tilde{u}_{\omega, \varsigma}(g), \quad g \in G .
\end{gathered}
$$

In particular, for 2-cocycle $\sigma_{\omega, \varsigma}, \tilde{\sigma}_{\omega, \varsigma}$ associated to $u_{\omega, \varsigma}, \tilde{u}_{\omega, \varsigma}$ respectively, we have $\left[\sigma_{\omega, \varsigma}\right]_{H^{2}(G, \mathbb{T})}=\left[\tilde{\sigma}_{\omega, \varsigma}\right]_{H^{2}(G, \mathbb{T})}$.

Proof. Let $\left(\mathcal{H}_{\omega_{\varsigma}}, \pi_{\omega_{\varsigma}}, \Omega_{\omega_{\varsigma}}\right)$ be the GNS triple of $\left.\omega\right|_{\mathcal{A}_{\varsigma}}$. The existence of irreducible *-representation $\left(\mathcal{L}_{\omega, \varsigma}, \rho_{\omega, \varsigma}\right)$ quasi-equivalent to $\pi_{\omega_{\varsigma}}$ follows from the definition of the split property.

To see the existence of $u_{\omega, \varsigma}$ satisfying (11) for such $\left(\mathcal{L}_{\omega, \varsigma}, \rho_{\omega, \varsigma}\right)$, let $\iota_{\omega, \varsigma}: \pi_{\omega_{\varsigma}}\left(\mathcal{A}_{\varsigma}\right)^{\prime \prime}$ $\rightarrow \mathcal{B}\left(\mathcal{L}_{\omega, \varsigma}\right)$ be the $*$-isomorphism such that $\rho_{\omega, \varsigma}=\iota_{\omega, \varsigma} \circ \pi_{\omega_{\varsigma}}$. By the $\tau_{\varsigma}$-invariance of $\left.\omega\right|_{\mathcal{A}_{\varsigma}}$, the action $\tau_{\varsigma}$ of $G$ can be extended to an action $\hat{\tau}_{\varsigma}$ on $\pi_{\omega_{\varsigma}}\left(\mathcal{A}_{\varsigma}\right)^{\prime \prime}$, so that $\hat{\tau}_{\varsigma}(g) \circ \pi_{\omega_{\varsigma}}=\pi_{\omega_{\varsigma}} \circ \tau_{\varsigma}(g)$, for $g \in G$. By the Wigner Theorem, the $*$-automorphism $\iota_{\omega, \varsigma} \circ \hat{\tau}_{\varsigma}(g) \circ \iota_{\omega, \varsigma}^{-1}$ on $\mathcal{B}\left(\mathcal{L}_{\omega, \varsigma}\right)$ is given by a unitary $u_{\omega, \varsigma}(g)$ so that

$$
\iota_{\omega, \varsigma} \circ \hat{\tau}_{\varsigma}(g) \circ \iota_{\omega, \varsigma}^{-1}=\operatorname{Ad}\left(u_{\omega, \varsigma}(g)\right), \quad g \in G .
$$


As $\hat{\tau}_{\varsigma}$ is an action of $G, u_{\omega, \varsigma}$ is a projective unitary representation. We obtain (11) by

$$
\begin{aligned}
\rho_{\omega, \varsigma} \circ \tau_{\varsigma}(g) & =\iota_{\omega, \varsigma} \circ \pi_{\omega_{\varsigma}} \circ \tau_{\varsigma}(g)=\iota_{\omega, \varsigma} \circ \hat{\tau}_{\varsigma}(g) \circ \pi_{\omega_{\varsigma}}=\iota_{\omega, \varsigma} \circ \hat{\tau}_{\varsigma}(g) \circ \iota_{\omega, \varsigma}^{-1} \circ \iota_{\omega, \varsigma} \circ \pi_{\omega_{\varsigma}} \\
& =\operatorname{Ad}\left(u_{\omega, \varsigma}(g)\right) \circ \rho_{\omega, \varsigma} .
\end{aligned}
$$

Suppose that $\left(\tilde{\mathcal{L}}_{\omega, \varsigma}, \tilde{\rho}_{\omega, \varsigma}, \tilde{u}_{\omega, \varsigma}\right)$ satisfies the same conditions as $\left(\mathcal{L}_{\omega, \varsigma}, \rho_{\omega, \varsigma}, u_{\omega, \varsigma}\right)$. Then by the Wigner Theorem, there exists a unitary $W: \mathcal{L}_{\omega, \varsigma} \rightarrow \tilde{\mathcal{L}}_{\omega, \varsigma}$ satisfying (12). Note that

$$
\begin{aligned}
\operatorname{Ad}\left(\tilde{u}_{\omega, \varsigma}(g)\right) \circ \tilde{\rho}_{\omega, \varsigma} & =\tilde{\rho}_{\omega, \varsigma} \circ \tau_{\varsigma}(g)=\operatorname{Ad} W \circ \rho_{\omega, \varsigma} \circ \tau_{\varsigma}(g) \\
& =\operatorname{Ad} W \circ \operatorname{Ad}\left(u_{\omega, \varsigma}(g)\right) \circ \operatorname{Ad} W^{*} \circ \tilde{\rho}_{\omega, \varsigma} .
\end{aligned}
$$

This implies that $\tilde{u}_{\omega, \varsigma}(g)^{*} \operatorname{Ad} W\left(u_{\omega, \varsigma}(g)\right)$ belongs to $\mathbb{T I}_{\tilde{\mathcal{L}}_{\omega, \varsigma}}$ proving (13).

Definition 1.7. Let $\omega \in S P G(\mathcal{A})$ and $\left(\mathcal{L}_{\omega, \varsigma}, \rho_{\omega, \varsigma}, u_{\omega, \varsigma}\right)$ be a triple satisfying the conditions in Proposition 1.6. Let $\sigma_{\omega, \varsigma}$ be the 2-cocycle associated to $u_{\omega, \varsigma}$. We call $\left(\mathcal{L}_{\omega, \varsigma}, \rho_{\omega, \varsigma}, u_{\omega, \varsigma}, \sigma_{\omega, \varsigma}\right)$ a quadruple associated to $\left(\left.\omega\right|_{\mathcal{A}_{\varsigma}}, \tau_{\varsigma}\right)$. Furthermore, we denote the second cohomology class $\left[\sigma_{\omega, \varsigma}\right]_{H^{2}(G, \mathbb{T})}$ by $c_{\omega, \zeta}$, and call it the second cohomology class of $G$ associated to $\left(\left.\omega\right|_{\mathcal{A}_{\varsigma}}, \tau_{\varsigma}\right)$.

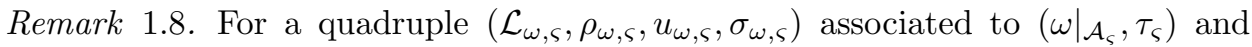
any map $b: G \rightarrow \mathbb{T},\left(\mathcal{L}_{\omega, \varsigma}, \rho_{\omega, \varsigma}, b \cdot u_{\omega, \varsigma}, \sigma_{b} \sigma_{\omega, \varsigma}\right)$ is also a quadruple associated to $\left(\left.\omega\right|_{\mathcal{A}_{\varsigma}}, \tau_{\varsigma}\right)$. See (4) and Notation 1.3 .

1.4. Main theorem. Let us introduce $\operatorname{AInn}^{G}\left(A_{\varsigma}\right)$.

Definition 1.9. Let $\varsigma=L, R$. An automorphism $\Xi_{\varsigma}$ of $\mathcal{A}_{\varsigma}$ is asymptotically inner in $\mathcal{A}_{\varsigma}^{G}$ if there is a norm continuous path $w_{\varsigma}:[0, \infty) \rightarrow \mathcal{U}\left(\mathcal{A}_{\varsigma}^{G}\right)$ with $w_{\varsigma}(0)=\mathbb{I}_{\mathcal{A}_{\varsigma}}$ that

$$
\Xi_{\varsigma}(a)=\lim _{t \rightarrow \infty} \operatorname{Ad}\left(w_{\varsigma}(t)\right)(a), \quad a \in \mathcal{A}_{\varsigma} .
$$

We denote by $\operatorname{AInn}^{G}\left(A_{\varsigma}\right)$ the set of all automorphisms which are asymptotically inner in $\mathcal{A}_{\varsigma}^{G}$.

For any pure states $\varphi_{1}$ and $\varphi_{2}$ on $\mathcal{A}$, there is an asymptotically inner automorphism $\Xi$ such that $\varphi_{2}=\varphi_{1} \circ \Xi$. This fact, which can be understood as a homogeneity of the pure states space, has been well-known from old time $[\mathrm{P}$, and after further development of the techniques and results, [B], FKK], the homogeneity is now proven for all the separable simple $C^{*}$-algebras in $[\mathrm{KOS}$.

In this paper, we consider the classification problem of $S P G(\mathcal{A})$ with respect to the following equivalence relation.

Definition 1.10. For $\omega_{0}, \omega_{1} \in S P G(\mathcal{A})$, we write $\omega_{0} \sim_{\text {split }, \tau} \omega_{1}$ if there exist automorphisms $\Xi_{L} \in \operatorname{AInn}^{G}\left(\mathcal{A}_{L}\right)$ and $\Xi_{R} \in \operatorname{AInn}^{G}\left(\mathcal{A}_{R}\right)$ such that $\omega_{1}$ and $\omega_{0} \circ$ $\left(\Xi_{L} \otimes \Xi_{R}\right)$ are quasi-equivalent.

Now we are ready to state our main theorem.

Theorem 1.11. For $\omega_{0}, \omega_{1} \in S P G(\mathcal{A}), \omega_{0} \sim_{\text {split }, \tau} \omega_{1}$ if and only if $c_{\omega_{1}, R}=c_{\omega_{0}, R}$. 
The "only if" part of the Theorem 1.11 is easy to prove. In order to prove "if" part of the Theorem, we note that if $c_{\omega_{1}, R}=c_{\omega_{0}, R}$ holds, $\omega_{0}$ and $\omega_{1}$ give covariant representations of a twisted $C^{*}$-dynamical systems $\Sigma_{\Gamma_{R}}^{\left(\sigma_{R}\right)}, \Sigma_{\Gamma_{L}}^{\left(\sigma_{L}\right)}$ (see section 3), where $\sigma_{R}, \sigma_{L}$ are 2-cocycles of $G$ such that $\left[\sigma_{R}\right]_{H^{2}(G, \mathbb{T})}=c_{\omega_{1}, R}=c_{\omega_{0}, R}$ and $\left[\sigma_{L}\right]_{H^{2}(G, \mathbb{T})}=c_{\omega_{1}, L}=c_{\omega_{0}, L}$. (See Remark 1.8 and Lemma 2.5.) One of the basic idea is to encode the information of these 2-cocycles $\sigma_{R}, \sigma_{L}$ into $C^{*}$-algebras we consider. Namely, instead of considering $\mathcal{A}_{R}, \mathcal{A}_{L}$, we consider the twisted crossed products $C^{*}\left(\Sigma_{\Gamma_{R}}^{\left(\sigma_{R}\right)}\right), C^{*}\left(\Sigma_{\Gamma_{L}}^{\left(\sigma_{L}\right)}\right)$. We recall the twisted crossed product $C^{*}\left(\Sigma_{\Gamma}^{(\sigma)}\right)$ of $\Sigma_{\Gamma}^{(\sigma)}$ in section 3. In section 2, we show that for any $\omega \in S P G(\mathcal{A})$, and $\varsigma=L, R, u_{\omega, \varsigma}$ contains all elements of $\mathcal{P}_{\sigma_{\omega, \varsigma}}$. Therefore, for any fixed $\alpha_{\varsigma} \in \mathcal{P}_{\sigma_{\varsigma}}$, both of $u_{\omega_{0}, \varsigma}$ and $u_{\omega_{1}, \varsigma}$ contains $\alpha_{\varsigma}$. This fact allows us to regard the problem as the homogeneity problem of $\mathcal{B}\left(\mathcal{H}_{\alpha_{\varsigma}}\right) \otimes C^{*}\left(\Sigma_{\Gamma_{\varsigma}}^{\left(\sigma_{\varsigma}\right)}\right)$, with symmetry (section 4). The proof of the homogeneity relies on the machinery developed in $[\mathrm{P},[\mathrm{B},[\mathrm{FKK}], \mathrm{KOS}]$. However, for our problem, we would like to take the path of unitaries in the fixed point algebras $\mathcal{A}_{R}^{G}, \mathcal{A}_{L}^{G}$. This requires some additional argument using the irreducible decompositions of $u_{\omega_{0}, \varsigma}, u_{\omega_{1}, \varsigma}$. This is given in section 4 ,

Before closing the introduction, let us remark on the relation between our result and the classification of SPT-phases. The notion of symmetry protected topological (SPT) phases was introduced by Gu and Wen GW]. It is defined as follows: we consider the set of all Hamiltonians with some symmetry, which have a unique gapped ground state in the bulk. We regard two of such Hamiltonians are equivalent, if there is a smooth path inside that family, connecting them. By this equivalence relation, we may classify the Hamiltonians in this family. A Hamiltonian which has only on-site interaction can be regarded as a trivial one. The set of Hamiltonians equivalent to such trivial ones represents a trivial phase. If a phase is nontrivial, it is a SPT phase. A basic question to ask is how to show that a given Hamiltonian belongs to a SPT phase. A mathematically natural approach for this problem is to define an invariant of the classification. An important fact for us in that context, which was proven by Matsui in [M2], is that the unique gapped ground states satisfy the split property. Hence there is a second cohomology class associated to symmetric gapped Hamiltonians. In [O], we showed that the second cohomology class is actually an invariant of the classification of SPT-phases. The question if it is a complete invariant is still open. Our classification criteria in this paper is coarser than the criterion of the classification of SPT-phases. However, it still follows the same philosophy as the original classification. The ground states in SPT-phases are states with short range entanglement, and as we saw in the beginning, the split states can be seen as states without macroscopic entanglement. And the operations we consider in our classification are the ones which preserves the entanglement property and the symmetry.

\section{IRREDUCIBLE COMPONENTS IN $u_{\omega, \varsigma}$}

In this section we show that $u_{\omega, \varsigma}$ contains all elements in $\mathcal{P}_{\sigma_{\omega, \varsigma}}$ with infinite multiplicity.

As $G$ is a finite group, its dual $\hat{G}$ is a finite set and we denote the number of the elements in $\hat{G}$ by $|\hat{G}|$. We use the following notation for any unitary/projective unitary representations $V_{1}, V_{2}$. We write $V_{1} \prec V_{2}$ if $V_{1}$ is unitarily equivalent to a 
sub-representation of $V_{2}$. We also say $V_{1}$ is included in $V_{2}$ in this case. Clearly, $\prec$ is a preorder. We write $V_{1} \cong V_{2}$ if $V_{1}$ and $V_{2}$ are unitarily equivalent.

For a unitary representation (resp. projective unitary representation) of $G$, we denote by $\bar{V}$ the complex conjugate representation (resp. projective representation) of $V$. (See [S] section II.6.)

Lemma 2.1. There is an $l_{0} \in \mathbb{N}$ such that for any $l \geq l_{0}$, the tensor product $U^{\otimes l}$ contains any irreducible representation of $G$ as its irreducible component.

Proof. Note that the character $\chi_{U}(g)$ is the sum of the eigenvalues of a unitary $U(g)$ acting on $\mathbb{C}^{d}$. Therefore, the maximal possible value of $\left|\chi_{U}(g)\right|$ is $d$, which is equal to $\chi_{U}(e)$. This value is attained only if $U(g) \in \mathbb{T I}_{\mathbb{C}^{d}}$. By the condition (2), for $g \in G \backslash\{e\},\left|\chi_{U}(g)\right|$ is strictly less than $d$.

Now for any irreducible representation $\left(\mathbb{C}^{m}, V\right)$ of $G$, for any $l \in \mathbb{N}$, we have

$$
\sum_{g \in G} \overline{\chi_{V}(g)} \chi_{U \otimes l}(g)=d^{l} \cdot m\left(1+\sum_{g \in G \backslash\{e\}} \frac{\overline{\chi_{V}(g)}}{m}\left(\frac{\chi_{U}(g)}{d}\right)^{l}\right) .
$$

Note that

$$
\overline{\frac{\chi_{V}(g)}{m}}\left(\frac{\chi_{U}(g)}{d}\right)^{l}
$$

for $g \in G \backslash\{e\}$ converges to 0 because of $\left|\chi_{U}(g)\right|<d$. Therefore, for $l$ large enough, the left hand side of (18) is non-zero. In other word, for $l$ large enough, $V$ is an irreducible component of $U^{\otimes l}$. As $\hat{G}$ is a finite set, (taking maximal such $l$ over all $V \in \hat{G})$, this proves the Lemma.

From this we obtain the following.

Lemma 2.2. There is an $l_{0} \in \mathbb{N}$ such that $\alpha \prec \beta \otimes U^{\otimes l}$ holds for any $\sigma \in Z^{2}(G, \mathbb{T})$, $\alpha, \beta \in \mathcal{P}_{\sigma}$, and $l_{0} \leq l \in \mathbb{N}$.

Proof. Let $l_{0}$ be the number given in Lemma 2.1. For any $\sigma \in Z^{2}(G, \mathbb{T})$ and $\alpha, \beta \in \mathcal{P}_{\sigma}, \alpha \otimes \bar{\beta}$ is a genuine representation of $G$. Let $V \in \hat{G}$ be an irreducible component of $\alpha \otimes \bar{\beta}$. By Lemma 2.1, this $V$ is realized as an irreducible component of $U^{\otimes l}$ for $l \geq l_{0}$. Therefore, for $l \geq l_{0}$, we have

$$
\sum_{g \in G} \overline{\chi_{\alpha}(g)} \chi_{\beta \otimes U \otimes l}(g)=\sum_{g \in G} \overline{\chi_{\alpha}(g) \chi_{\bar{\beta}}(g)} \chi_{U \otimes l}(g) \geq \sum_{g \in G} \overline{\chi_{V}(g)} \chi_{U \otimes l}(g)>0 .
$$

This means $\alpha \prec \beta \otimes U^{\otimes l}$.

Lemma 2.3. Let $\sigma \in Z^{2}(G, \mathbb{T})$ be a fixed 2-cocycle. For any $m \in \mathbb{N}$, there exists an $N_{m}^{(\sigma)} \in \mathbb{N}$ satisfying the following: For any projective unitary representation $(\mathcal{H}, u)$ of $G$ with 2-cocycle $\sigma, \alpha \in \mathcal{P}_{\sigma}$, and $\mathbb{N} \ni N \geq N_{m}^{(\sigma)}$, we have

$$
m \cdot \alpha \prec U^{\otimes N} \otimes u \text {. }
$$

(Here $m \cdot \alpha$ denotes the $m$ direct sum of $\alpha$.)

Proof. First let us consider the case that $\mathcal{P}_{\sigma}$ consists of a unique element $\alpha \in \mathcal{P}_{\sigma}$. Then for any $N \in \mathbb{N}$, and any projective representation $(\mathcal{H}, u, \sigma)$, the multiplicity of $\alpha$ in $U^{\otimes N} \otimes u$ is $\frac{d^{N} \cdot \operatorname{dim} \mathcal{H}}{n_{\alpha}}$, which is bigger or equal to a $(\mathcal{H}, u)$-independent value $\frac{d^{N}}{n_{\alpha}}$. The claim of Lemma 2.3 follows from this immediately for this case. 
Next let us consider the case that the number of elements $\left|\mathcal{P}_{\sigma}\right|$ in $\mathcal{P}_{\sigma}$, is larger than 1. From Lemma 2.2, choose $l_{0} \in \mathbb{N}$ so that $\alpha \prec \beta \otimes U^{\otimes l}$ for all $l \geq l_{0}$ and $\alpha, \beta \in \mathcal{P}_{\sigma}$. For any $m \in \mathbb{N}$, choose $M_{m} \in \mathbb{N}$ so that $\left|\mathcal{P}_{\sigma}\right|^{M_{m}}>m$. Here we use the condition that $\left|\mathcal{P}_{\sigma}\right|>1$. We set $N_{m}^{(\sigma)}:=l_{0}\left(M_{m}+1\right)$. Let $(\mathcal{H}, u)$ be a projective unitary representation of $G$ with 2-cocycle $\sigma, \alpha \in \mathcal{P}_{\sigma}$, and $\mathbb{N} \ni N \geq N_{m}^{(\sigma)}$. We would like to show that $m \cdot \alpha \prec U^{\otimes N} \otimes u$. By the choice of $N_{m}^{(\sigma)}, N$ can be decomposed as $N=k_{1}+k_{2}+\cdots+k_{M_{m}}+k_{M_{m}+1}$ with some $l_{0} \leq k_{j} \in \mathbb{N}, j=1, \ldots, M_{m}+1$. For each $j=1, \ldots, M_{m}+1$ and $\beta, \gamma \in \mathcal{P}_{\sigma}$, we denote the multiplicity of $\gamma$ in $U^{\otimes k_{j}} \otimes \beta$ by $n_{\beta, \gamma}^{(j)}$. From the choice of $l_{0}$, we have $1 \leq n_{\beta, \gamma}^{(j)}$ for any $j=1, \ldots, M_{m}+1$ and $\beta, \gamma \in \mathcal{P}_{\sigma}$. Fix some $\beta_{0} \in \mathcal{P}_{\sigma}$ such that $\beta_{0} \prec u$. From this, we get

(22) $m \cdot \alpha \prec\left|\mathcal{P}_{\sigma}\right|^{M_{m}} \cdot \alpha$

$$
\begin{aligned}
& =\bigoplus_{\gamma_{1}, \gamma_{2}, \ldots, \gamma_{M_{m}}} \alpha \prec \underset{\gamma_{1}, \gamma_{2}, \ldots, \gamma_{M_{m}}, \gamma_{M_{m}+1}}{\prec} n_{\beta_{0}, \gamma_{1}}^{(1)} n_{\gamma_{1}, \gamma_{2}}^{(2)} \cdots n_{\gamma_{M_{m}}, \gamma_{M_{m}+1}}^{\left(M_{m}+1\right)} \cdot \gamma_{M_{m}+1} \\
& \prec U^{\otimes k_{M_{m}+1}} \otimes U^{\otimes k_{M_{m}}} \otimes \cdots U^{\otimes k_{2}} \otimes U^{\otimes k_{1}} \otimes \beta_{0}=U^{\otimes N} \otimes \beta_{0} \prec U^{\otimes N} \otimes u .
\end{aligned}
$$

This completes the proof.

Now we are ready to show the main statement of this section. From the following Lemma, we see that for any $\omega \in S P G(\mathcal{A}), u_{\omega, \varsigma}$ contains all elements of $\mathcal{P}_{\sigma_{\omega, \varsigma}}$ with infinite multiplicity.

Theorem 2.4. Let $\Gamma$ be an infinite subset of $\mathbb{Z}$. Let $(\mathcal{L}, \rho, u, \sigma)$ be a quadruple such that

(i): $\rho$ is a $*$-representation of $\mathcal{A}_{\Gamma}$ on a Hilbert space $\mathcal{L}$,

(ii): $u$ is a projective unitary representation of $G$ on $\mathcal{L}$ with a 2-cocycle $\sigma$,

(iii): for any $g \in G$, we have

$$
\rho \circ \tau_{\Gamma}(g)=\operatorname{Ad}(u(g)) \circ \rho .
$$

Then $u$ contains all elements of $\mathcal{P}_{\sigma}$ with infinite multiplicity.

Proof. Fix any $\alpha \in \mathcal{P}_{\sigma}$ and $m \in \mathbb{N}$. We would like to show that $m \cdot \alpha \prec u$. Let $N_{m}^{(\sigma)}$ be the number given in Lemma 2.3 for this fixed $m$. Let $\Lambda$ be a subset of $\Gamma$ such that $|\Lambda|=N_{m}^{(\sigma)}$. We may factorize $(\mathcal{L}, \rho, u)$ to $\Lambda$-part and $\Gamma \backslash \Lambda$-part as follows: There exist a $*$-representation $(\tilde{\mathcal{L}}, \tilde{\rho})$ of $\mathcal{A}_{\Gamma \backslash \Lambda}$ and a projective unitary representation $\tilde{u}$ of $G$ on $\tilde{\mathcal{L}}$ with 2 -cocycle $\sigma$, implementing $\tau_{\Gamma \backslash \Lambda}$. There exists a unitary $W: \mathcal{L} \rightarrow\left(\otimes_{\Lambda} \mathbb{C}^{d}\right) \otimes \tilde{\mathcal{L}}$ such that

$$
W \rho(a) W^{*}=\left(\operatorname{id}_{\mathcal{A}_{\Lambda}} \otimes \tilde{\rho}\right)(a), \quad a \in \mathcal{A}_{\Gamma},
$$

and

$$
W u(g) W^{*}=\left(\bigotimes_{\Lambda} U(g)\right) \otimes \tilde{u}(g), \quad g \in G .
$$

More precisely, set $I_{0}=\left(i_{k}\right)_{k \in \Lambda} \in\{1, \ldots, d\}^{\times \Lambda}$, with $i_{k}=1$ for all $k \in \Lambda$. We define the Hilbert space $\tilde{\mathcal{L}}$ by $\tilde{\mathcal{L}}:=\rho\left(E_{I_{0}, I_{0}}^{(\Lambda)}\right) \mathcal{L}$, and the $*$-representation $\tilde{\rho}$ of $\mathcal{A}_{\Gamma \backslash \Lambda}$ on $\tilde{\mathcal{L}}$ by

$$
\tilde{\rho}(a):=\rho\left(E_{I_{0}, I_{0}}^{(\Lambda)} \otimes a\right), \quad a \in \mathcal{A}_{\Gamma \backslash \Lambda} .
$$


The unitary $W: \mathcal{L} \rightarrow\left(\otimes_{\Lambda} \mathbb{C}^{d}\right) \otimes \tilde{\mathcal{L}}$ is defined by

$$
W \xi:=\sum_{I \in\{1, \ldots, n\} \times \Lambda} e_{I}^{(\Lambda)} \otimes \rho\left(E_{I_{0}, I}^{(\Lambda)}\right) \xi, \quad \xi \in \mathcal{L} .
$$

It is straight forward to check (24).

By a straight forward calculation using (24), we can check that

$$
\left(\left(\bigotimes_{\Lambda} U(g)\right)^{*} \otimes \mathbb{I}_{\tilde{\mathcal{L}}}\right) W u(g) W^{*}
$$

with $g \in G$ commute with any element of $\left(\bigotimes_{\Lambda} \mathrm{M}_{d}\right) \otimes \mathbb{C I}_{\tilde{\mathcal{L}}}$. Hence there exists

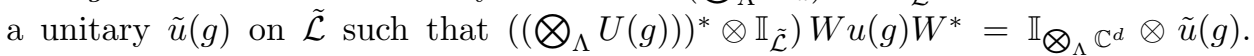
This gives (25). It is straight forward to check that $\tilde{u}$ is a projective unitary representation of $G$ with 2-cocycle $\sigma$ implementing $\tau_{\Gamma \backslash \Lambda}$.

From (25) and Lemma 2.3, we have

$$
m \cdot \alpha \prec U^{\otimes N_{m}^{(\sigma)}} \otimes \tilde{u}=U^{\otimes \Lambda} \otimes \tilde{u} \cong u .
$$

This completes the proof.

Recall Definition 1.7. We note that $c_{\omega, R}$ and $c_{\omega, L}$ are not independent.

Lemma 2.5. For any $\omega \in S P G(\mathcal{A})$, we have $c_{\omega, R}=c_{\omega, L}^{-1}$.

Proof. Let $(\mathcal{H}, \pi, \Omega)$ be the GNS triple of $\omega$. As $\omega$ satisfies the split property, there are Hilbert spaces $\mathcal{H}_{L}, \mathcal{H}_{R}$ and a unitary $W: \mathcal{H} \rightarrow \mathcal{H}_{L} \otimes \mathcal{H}_{R}$ such that

$$
W \pi\left(\mathcal{A}_{R}\right)^{\prime \prime} W^{*}=\mathbb{C I}_{\mathcal{H}_{L}} \otimes \mathcal{B}\left(\mathcal{H}_{R}\right), \quad W \pi\left(\mathcal{A}_{R}\right)^{\prime} W^{*}=\mathcal{B}\left(\mathcal{H}_{L}\right) \otimes \mathbb{C I}_{\mathcal{H}_{R}} .
$$

(See Theorem 1.31 V [T].) From (29), $\pi\left(\mathcal{A}_{L}\right)^{\prime \prime} \subset \pi\left(\mathcal{A}_{R}\right)^{\prime}$ and $\pi\left(\mathcal{A}_{L}\right)^{\prime \prime} \vee \pi\left(\mathcal{A}_{R}\right)^{\prime \prime}=$ $\mathcal{B}(\mathcal{H})$, we obtain

$$
W \pi\left(\mathcal{A}_{L}\right)^{\prime \prime} W^{*}=\mathcal{B}\left(\mathcal{H}_{L}\right) \otimes \mathbb{C I}_{\mathcal{H}_{R}} .
$$

Hence we obtain irreducible representations $\left(\mathcal{H}_{L}, \pi_{L}\right)$ and $\left(\mathcal{H}_{R}, \pi_{R}\right)$ of $\mathcal{A}_{L}, \mathcal{A}_{R}$ such that

$$
W \pi(a \otimes b) W^{*}=\pi_{L}(a) \otimes \pi_{R}(b), \quad a \in \mathcal{A}_{L}, \quad b \in \mathcal{A}_{R} .
$$

The triple $\left(\mathcal{H}_{L} \otimes \mathcal{H}_{R}, \pi_{L} \otimes \pi_{R}, W \Omega\right)$ is a GNS triple of $\omega$. Therefore, $\left.\omega\right|_{\mathcal{A}_{R}}$ is $\pi_{R}$-normal. As $\pi_{R}\left(\mathcal{A}_{R}\right)^{\prime \prime}$ is a factor, $\pi_{R}$ and the GNS representation of $\left.\omega\right|_{\mathcal{A}_{R}}$ are quasi-equivalent. Similarly, $\pi_{L}$ and the GNS representation of $\left.\omega\right|_{\mathcal{A}_{L}}$ are quasiequivalent.

By the $\tau$-invariance of $\omega$, there is a unitary representation $V$ of $G$ on $\mathcal{H}_{L} \otimes \mathcal{H}_{R}$ given by

$$
V(g)\left(\pi_{L} \otimes \pi_{R}\right)(a) W \Omega=\left(\pi_{L} \otimes \pi_{R}\right)(\tau(g)(a)) W \Omega, \quad g \in G, \quad a \in \mathcal{A} .
$$

On the other hand, by Proposition 1.6, there are projective unitary representations $u_{L}, u_{R}$ of $G$ on $\mathcal{H}_{L}, \mathcal{H}_{R}$ such that

(33) $\pi_{L} \circ \tau_{L}(g)(a)=\operatorname{Ad}\left(u_{L}(g)\right) \circ \pi_{L}(a), \quad \pi_{R} \circ \tau_{R}(g)(b)=\operatorname{Ad}\left(u_{R}(g)\right) \circ \pi_{R}(b)$, for all $a \in \mathcal{A}_{L}, b \in \mathcal{A}_{R}$ and $g \in G$. Note that

$$
\begin{aligned}
\operatorname{Ad}(V(g)) \circ\left(\pi_{L} \otimes \pi_{R}\right)(x) & =\left(\pi_{L} \otimes \pi_{R}\right) \circ \tau(g)(x) \\
& =\operatorname{Ad}\left(u_{L}(g) \otimes u_{R}(g)\right) \circ\left(\pi_{L} \otimes \pi_{R}\right)(x),
\end{aligned}
$$


for all $x \in \mathcal{A}$. As $\left(\pi_{L} \otimes \pi_{R}\right)(\mathcal{A})^{\prime \prime}=\mathcal{B}\left(\mathcal{H}_{L} \otimes \mathcal{H}_{R}\right)$, this means that there is a map $b: G \rightarrow \mathbb{T}$ such that

$$
u_{L}(g) \otimes u_{R}(g)=b(g) V(g), \quad g \in G .
$$

Let $\sigma_{L}, \sigma_{R} \in \mathbb{Z}^{2}(G, \mathbb{T})$ be 2-cocycles of $u_{L}, u_{R}$ respectively. From (35), we obtain

$$
\sigma_{L} \sigma_{R}=\sigma_{b}
$$

(Here $\sigma_{b}$ is defined by (4).) This means

$$
c_{\omega, R}=\left[\sigma_{R}\right]_{H^{2}(G, \mathbb{T})}=\left[\sigma_{L}^{-1}\right]_{H^{2}(G, \mathbb{T})}=c_{\omega, L}^{-1} .
$$

\section{TWisted $C^{*}$-DYNAMICAL SYSTEM}

In this section we briefly recall basic facts about twisted $C^{*}$-crossed product. Throughout this section, let $\Gamma$ be an infinite subset of $\mathbb{Z}$, and $\sigma \in Z^{2}(G, \mathbb{T})$. The quadruple $\left(G, \mathcal{A}_{\Gamma}, \tau_{\Gamma}, \sigma\right)$ is a twisted $C^{*}$-dynamical system which we denote by $\Sigma_{\Gamma}^{(\sigma)}$. (This is a simple version of $[\mathrm{BC}$.)

A covariant representation of $\Sigma_{\Gamma}^{(\sigma)}$ is a triple $(\mathcal{H}, \pi, u)$ where $\pi$ is a $*$-representation of the $C^{*}$-algebra $\mathcal{A}_{\Gamma}$ on a Hilbert space $\mathcal{H}$ and $u$ is a projective unitary representation of $G$ with 2-cocycle $\sigma$ on $\mathcal{H}$ such that

$$
u(g) \pi(a) u(g)^{*}=\pi\left(\tau_{\Gamma}(g)(a)\right), \quad a \in \mathcal{A}_{\Gamma}, \quad g \in G .
$$

In this paper, we say the covariant representation $(\mathcal{H}, \pi, u)$ is irreducible if $\pi$ is an irreducible representation of $\mathcal{A}_{\Gamma}$. Note that for a quadruple $\left(\mathcal{L}_{\omega, \varsigma}, \rho_{\omega, \varsigma}, u_{\omega, \varsigma}, \sigma_{\omega, \varsigma}\right)$ associated to $\left(\left.\omega\right|_{\mathcal{A}_{\varsigma}}, \tau_{\varsigma}\right)$ with $\omega \in S P G(\mathcal{A})$ (Definition 1.7), $\left(\mathcal{L}_{\omega, \varsigma}, \rho_{\omega, \varsigma}, u_{\omega, \varsigma}\right)$ is an irreducible covariant representation of $\Sigma_{\Gamma_{\varsigma}}^{\left(\sigma_{\omega, \varsigma}\right)}$.

Let $C\left(G, \mathcal{A}_{\Gamma}\right)$ be the linear space of $\mathcal{A}_{\Gamma}$-valued functions on $G$. We equip $C\left(G, \mathcal{A}_{\Gamma}\right)$ with a product and $*$-operation as follows:

$$
\begin{aligned}
& f_{1} * f_{2}(h):=\sum_{g \in G} \sigma\left(g, g^{-1} h\right) \cdot f_{1}(g) \cdot \tau_{\Gamma}(g)\left(f_{2}\left(g^{-1} h\right)\right), \quad h \in G, \\
& f^{*}(h):=\overline{\sigma\left(h^{-1}, h\right)} \tau_{\Gamma}(h)\left(f\left(h^{-1}\right)^{*}\right), \quad h \in G,
\end{aligned}
$$

for $f_{1}, f_{2}, f \in C\left(G, \mathcal{A}_{\Gamma}\right)$. The linear space $C\left(G, \mathcal{A}_{\Gamma}\right)$ which is a $*$-algebra with these operations is denoted by $C\left(\Sigma_{\Gamma}^{(\sigma)}\right)$. We will omit the symbol $*$ for the multiplication (39).

For a covariant representation $(\mathcal{H}, \pi, u)$ of $\Sigma_{\Gamma}^{(\sigma)}$, we may introduce a *-representation $(\mathcal{H}, \pi \times u)$ of $C\left(\Sigma_{\Gamma}^{(\sigma)}\right)$ by

$$
(\pi \times u)(f):=\sum_{g \in G} \pi(f(g)) u(g), \quad f \in C\left(\Sigma_{\Gamma}^{(\sigma)}\right) .
$$

The full twisted crossed product of $\Sigma_{\Gamma}^{(\sigma)}$, denoted $C^{*}\left(\Sigma_{\Gamma}^{(\sigma)}\right)$ is the completion of $C\left(\Sigma_{\Gamma}^{(\sigma)}\right)$ with respect to the norm

(42) $\|f\|_{u}:=\sup \{\|(\pi \times u)(f)\| \mid(\pi, u):$ covariant representation $\}, \quad f \in C\left(\Sigma_{\Gamma}^{(\sigma)}\right)$.

From any representation $(\mathcal{H}, \pi)$ of $\mathcal{A}_{\Gamma}$, we can define a covariant representation $\left(\mathcal{H} \otimes l^{2}(G) \simeq l^{2}(G, \mathcal{H}), \tilde{\pi}, \tilde{u}_{\pi}\right)$ of $\Sigma_{\Gamma}^{(\sigma)}$ by

$$
(\tilde{\pi}(a) \xi)(g):=\pi\left(\tau_{\Gamma}\left(g^{-1}\right)(a)\right) \xi(g), \quad a \in \mathcal{A}_{\Gamma}, \quad \xi \in l^{2}(G, \mathcal{H}), \quad g \in G,
$$


and $\tilde{u}_{\pi}=\mathbb{I}_{\mathcal{H}} \otimes u_{r}^{\sigma}$. Here, $u_{r}^{\sigma}$ is a projective unitary representation with 2-cocycle $\sigma$ on $l^{2}(G)$ defined by

$$
\left(u_{r}^{\sigma}(g) \xi\right)(h)=\sigma\left(g, g^{-1} h\right) \xi\left(g^{-1} h\right), \quad g, h \in G, \quad \xi \in l^{2}(G) .
$$

Note that $\pi$ is faithful because $\mathcal{A}_{\Gamma}$ is simple. Therefore, the representation $\tilde{\pi} \times \tilde{u}_{\pi}$ of $C\left(\Sigma_{\Gamma}^{(\sigma)}\right)$ given by $\left(\mathcal{H} \otimes l^{2}(G) \simeq l^{2}(G, \mathcal{H}), \tilde{\pi}, \tilde{u}_{\pi}\right)$ is faithful. We define a $C^{*}$-norm $\|\cdot\|_{r}$ on $C\left(\Sigma_{\Gamma}^{(\sigma)}\right)$ by

$$
\|f\|_{r}:=\left\|\tilde{\pi} \times \tilde{u}_{\pi}(f)\right\|_{\mathcal{B}\left(\mathcal{H} \otimes l^{2}(G)\right)}, \quad f \in C\left(\Sigma_{\Gamma}^{(\sigma)}\right) .
$$

The completion $C_{r}^{*}\left(\Sigma_{\Gamma}^{(\sigma)}\right)$ of $C\left(\Sigma_{\Gamma}^{(\sigma)}\right)$ with respect to this norm is the reduced twisted crossed product of $\Sigma_{\Gamma}^{(\sigma)}$. As we are considering a finite group $G$, we have $C\left(\Sigma_{\Gamma}^{(\sigma)}\right)=$ $C_{r}^{*}\left(\Sigma_{\Gamma}^{(\sigma)}\right)=C^{*}\left(\Sigma_{\Gamma}^{(\sigma)}\right)$, and $\|\cdot\|_{r}=\|\cdot\|_{u}$. Hereafter, we will use $f_{1} f_{2}$ instead of the convolution notation $f_{1} * f_{2}$ to represent the multiplication of $f_{1}$ and $f_{2}$ in $C^{*}\left(\Sigma_{\Gamma}\right)$.

For each $a \in \mathcal{A}_{\Gamma}, \xi_{a}: G \ni g \mapsto \delta_{g, e} a \in \mathcal{A}_{\Gamma}$ defines an element of $C^{*}\left(\Sigma_{\Gamma}^{(\sigma)}\right)$. The map $\xi: \mathcal{A}_{\Gamma} \ni a \mapsto \xi_{a} \in C^{*}\left(\Sigma_{\Gamma}^{(\sigma)}\right)$ is a unital faithful $*$-homomorphism. Note that $\xi_{\mathbb{I}_{\mathcal{A}_{\Gamma}}}$ is the identity of $C^{*}\left(\Sigma_{\Gamma}^{(\sigma)}\right)$. Hence the $C^{*}$-algebra $\mathcal{A}_{\Gamma}$ can be regarded as a subalgebra of $C\left(\Sigma_{\Gamma}^{(\sigma)}\right)=C_{r}^{*}\left(\Sigma_{\Gamma}^{(\sigma)}\right)=C^{*}\left(\Sigma_{\Gamma}^{(\sigma)}\right)$. Therefore, we simply write $a$ to denote $\xi_{a}$.

From the condition (2), for any $g \in G$ with $g \neq e$, the automorphism $\tau_{\Gamma}(g)$ is properly outer. Therefore, by the argument in $[\mathrm{E}]$ Theorem $3.2, C\left(\Sigma_{\Gamma}^{(\sigma)}\right)=$ $C_{r}^{*}\left(\Sigma_{\Gamma}^{(\sigma)}\right)=C^{*}\left(\Sigma_{\Gamma}^{(\sigma)}\right)$ is simple.

As $\mathcal{A}_{\Gamma}$ is unital, we have unitaries $\lambda_{g} \in C^{*}\left(\Sigma_{\Gamma}^{(\sigma)}\right), g \in G$, defined by $G \ni h \mapsto$ $\delta_{g, h} \mathbb{I}_{\mathcal{A}_{\Gamma}} \in \mathcal{A}_{\Gamma}$ such that

$$
\begin{array}{ll}
\lambda_{g} \lambda_{h}=\sigma(g, h) \lambda_{g h}, & g, h \in G, \\
\lambda_{g} a \lambda_{g}^{*}=\tau_{\Gamma}(g)(a), & a \in \mathcal{A}_{\Gamma}, g \in G .
\end{array}
$$

Note that $\lambda_{e}=\xi_{\mathbb{A}_{\mathcal{A}_{\Gamma}}}$ is the identity of $C^{*}\left(\Sigma_{\Gamma}^{(\sigma)}\right)$.

We set

$$
C^{*}\left(\Sigma_{\Gamma}^{(\sigma)}\right)^{G}:=\left\{f \in C^{*}\left(\Sigma_{\Gamma}^{(\sigma)}\right) \mid \operatorname{Ad}\left(\lambda_{g}\right)(f)=f, \quad g \in G\right\} .
$$

Let $(\mathcal{H}, \pi, u)$ be an irreducible covariant representation of $\Sigma_{\Gamma}^{(\sigma)}$. The projective unitary representation $u$ has an irreducible decomposition given by some Hilbert spaces $\left\{\mathcal{K}_{\gamma} \mid \gamma \in \mathcal{P}_{\sigma}\right\}$ (Lemma 1.1 and Notation 1.2). Namely we have

$$
u(g)=\bigoplus_{\alpha \in \mathcal{P}_{\sigma}} V_{\alpha}(g) \otimes \mathbb{I}_{\mathcal{K}_{\alpha}}, \quad g \in G, \quad \text { and } \quad u(G)^{\prime}=\bigoplus_{\alpha \in \mathcal{P}_{\sigma}} \mathbb{I}_{\mathcal{H}_{\alpha}} \otimes \mathcal{B}\left(\mathcal{K}_{\alpha}\right) .
$$

Note that

$$
(\pi \times u)\left(\lambda_{g}\right)=u(g), \quad g \in G
$$

From this we have

$$
(\pi \times u)\left(C^{*}\left(\Sigma_{\Gamma}^{(\sigma)}\right)^{G}\right) \subset u(G)^{\prime}=\bigoplus_{\alpha \in \mathcal{P}_{\sigma}} \mathbb{C I}_{\mathcal{H}_{\alpha}} \otimes \mathcal{B}\left(\mathcal{K}_{\alpha}\right) .
$$

The following proposition is the immediate consequence of Theorem 2.4 
Proposition 3.1. Let $(\mathcal{H}, \pi, u)$ be an irreducible covariant representation of $\Sigma_{\Gamma}^{(\sigma)}$. Then $u$ contains all elements of $\mathcal{P}_{\sigma}$ with infinite multiplicity.

\section{Homogeneity}

Throughout this section we fix $\Gamma=\Gamma_{L}, \Gamma_{R}, \sigma \in Z^{2}(G, \mathbb{T})$, and $\alpha \in \mathcal{P}_{\sigma}$. We use the following notation.

Notation 4.1. Let $(\mathcal{H}, \pi, u)$ be an irreducible covariant representation of $\Sigma_{\Gamma}^{(\sigma)}$ with an irreducible decomposition of $u$ given by a set of Hilbert spaces $\left\{\mathcal{K}_{\gamma} \mid \gamma \in \mathcal{P}_{\sigma}\right\}$. We use the symbol $\hat{\pi}$ to denote the irreducible representation

$$
\hat{\pi}=\operatorname{id}_{\mathcal{B}\left(\mathcal{H}_{\alpha}\right)} \otimes(\pi \times u)
$$

of $\mathcal{B}\left(\mathcal{H}_{\alpha}\right) \otimes C^{*}\left(\Sigma_{\Gamma}^{(\sigma)}\right)$ on $\mathcal{H}_{\alpha} \otimes \mathcal{H}$.

For a unit vector $\xi \in \mathcal{K}_{\alpha}$, we may define a state $\hat{\varphi}_{\xi}$ on $\mathcal{B}\left(\mathcal{H}_{\alpha}\right) \otimes C^{*}\left(\Sigma_{\Gamma}^{(\sigma)}\right)$ by

$$
\hat{\varphi}_{\xi}(x):=\langle\tilde{\xi}, \hat{\pi}(x) \tilde{\xi}\rangle, \quad x \in \mathcal{B}\left(\mathcal{H}_{\alpha}\right) \otimes C^{*}\left(\Sigma_{\Gamma}^{(\sigma)}\right) .
$$

Here, $\tilde{\xi}$ is an element of $\mathcal{H}_{\alpha} \otimes \mathcal{H}$,

$$
\tilde{\xi}:=\Omega_{\alpha} \otimes \xi \in \mathcal{H}_{\alpha} \otimes \mathcal{H}_{\alpha} \otimes \mathcal{K}_{\alpha} \hookrightarrow \mathcal{H}_{\alpha} \otimes \mathcal{H}
$$

regarding $\mathcal{H}_{\alpha} \otimes \mathcal{H}_{\alpha} \otimes \mathcal{K}_{\alpha}$ as a subspace of $\mathcal{H}_{\alpha} \otimes \mathcal{H}$. (See Notation 1.2.) Recall that $\Omega_{\alpha}$ is defined in (6). We call this $\hat{\varphi}_{\xi}$ a state on $\mathcal{B}\left(\mathcal{H}_{\alpha}\right) \otimes C^{*}\left(\Sigma_{\Gamma}^{(\sigma)}\right)$ given by $(\mathcal{H}, \pi, u, \xi)$. By the irreducibility of $\pi, \hat{\pi}$ is irreducible and $\hat{\varphi}_{\xi}$ is a pure state on $\mathcal{B}\left(\mathcal{H}_{\alpha}\right) \otimes C^{*}\left(\Sigma_{\Gamma}^{(\sigma)}\right)$. Note that $\left(\mathcal{H}_{\alpha} \otimes \mathcal{H}, \hat{\pi}, \tilde{\xi}\right)$ is a GNS triple of $\hat{\varphi}_{\xi}$.

The goal of this section is to prove the following Proposition.

Proposition 4.2. Let $\left(\mathcal{H}_{i}, \pi_{i}, u_{i}\right)$ with $i=0,1$ be irreducible covariant representations of $\Sigma_{\Gamma}^{(\sigma)}$ with irreducible decomposition of $u_{i}$ given by a set of Hilbert spaces $\left\{\mathcal{K}_{\gamma, i} \mid \gamma \in \mathcal{P}_{\sigma}\right\}$. Let $\xi_{i} \in \mathcal{K}_{\alpha, i}$ be unit vectors in $\mathcal{K}_{\alpha, i}$ for $i=0,1$. (Recall Proposition 3.1 for existence of such vectors.) Let $\hat{\varphi}_{\xi_{i}}$ be a state on $\mathcal{B}\left(\mathcal{H}_{\alpha}\right) \otimes C^{*}\left(\Sigma_{\Gamma}^{(\sigma)}\right)$ given by $\left(\mathcal{H}_{i}, \pi_{i}, u_{i}, \xi_{i}\right)$, for each $i=0,1$. Let $\varphi_{i}$ be the restriction of $\hat{\varphi}_{\xi_{i}}$ onto $\mathcal{A}_{\Gamma}$. Then there exists a norm-continuous path $w:[0, \infty) \rightarrow \mathcal{U}\left(\mathcal{A}_{\Gamma}^{G}\right)$ with $w(0)=\mathbb{I}$, such that

(1) for each $a \in \mathcal{A}_{\Gamma}$, the limit

$$
\lim _{t \rightarrow \infty} \operatorname{Ad}(w(t))(a)=: \Xi_{\Gamma}(a)
$$

exists and defines an automorphism $\Xi_{\Gamma}$ on $\mathcal{A}_{\Gamma}$, and

(2) the automorphism $\Xi_{\Gamma}$ in 1. satisfies $\varphi_{1}=\varphi_{0} \circ \Xi_{\Gamma}$.

Remark 4.3. Basically, what we would like to do is to connect some $\pi_{0}$-normal state $\varphi_{0}$ and some $\pi_{1}$-normal state $\varphi_{1}$ via some $\Xi_{\Gamma} \in \operatorname{AInn}^{G}\left(A_{\Gamma}\right)$. Without symmetry, $\varphi_{0}$ and $\varphi_{1}$ can be taken to be pure states. When the symmetry comes into the game, to guarantee that $\Xi_{\Gamma}$ commutes with $\tau_{\Gamma}(g)$, we would like to assume that $\varphi_{0}$ and $\varphi_{1}$ are $\tau_{\Gamma}$-invariant. If $\sigma$ is trivial, there is a $u_{i}$-invariant non-zero vector that we may find such pure $\tau_{\Gamma}$-invariant states $\varphi_{0}$ and $\varphi_{1}$. But if the cohomology class of $\sigma$ is not trivial, $u_{i}$ does not have a non-zero invariant vector. However, there is still a rank $n_{\alpha} u_{i}$-invariant density matrix. That is the reason why we consider $\mathcal{B}\left(\mathcal{H}_{\alpha}\right) \otimes C^{*}\left(\Sigma_{\Gamma}^{(\sigma)}\right)$. Note that the density matrix of $\varphi_{i}$ is a rank $n_{\alpha}$ operator which commutes with $u_{i}$. 
For the proof of Proposition 4.2, we use the machinery used in [FKK] and [KOS]. (See Appendix B.) However, as we would like to have a path in the fixed point algebra $\mathcal{A}_{\Gamma}^{G}$, we need additional arguments. For that purpose, the following Lemma plays an important role.

Lemma 4.4. Let $\Gamma_{0}$ be an infinite subset of $\mathbb{Z}$. Let $(\mathcal{H}, \pi, u)$ be an irreducible covariant representation of $\Sigma_{\Gamma_{0}}^{(\sigma)}$ with an irreducible decomposition of u given by a set of Hilbert spaces $\left\{\mathcal{K}_{\gamma} \mid \gamma \in \mathcal{P}_{\sigma}\right\}$. Then there exist irreducible *-representations $\left(\mathcal{K}_{\gamma}, \pi_{\gamma}\right), \gamma \in \mathcal{P}_{\sigma}$ of $\mathcal{A}_{\Gamma_{0}}^{G}$ such that

$$
\pi(a)=\bigoplus_{\gamma \in \mathcal{P}_{\sigma}} \mathbb{I}_{\mathcal{H}_{\gamma}} \otimes \pi_{\gamma}(a), \quad a \in \mathcal{A}_{\Gamma_{0}}^{G} .
$$

Furthermore, we have

$$
\pi\left(\mathcal{A}_{\Gamma_{0}}^{G}\right)^{\prime \prime}=\bigoplus_{\gamma \in \mathcal{P}_{\sigma}} \mathbb{I}_{\mathcal{H}_{\gamma}} \otimes B\left(\mathcal{K}_{\gamma}\right) .
$$

Notation 4.5. We call $\left\{\left(\mathcal{K}_{\gamma}, \pi_{\gamma}\right) \mid \gamma \in \mathcal{P}_{\sigma}\right\}$, the family of representations of $\mathcal{A}_{\Gamma_{0}}^{G}$ associated to $(\mathcal{H}, \pi, u)$.

Proof. For any $a \in \mathcal{A}_{\Gamma_{0}}^{G}$, we have $\pi(a) \in u(G)^{\prime}$. Therefore, from Lemma 1.1, each $\pi(a)$ with $a \in \mathcal{A}_{\Gamma_{0}}^{G}$ has a form

$$
\pi(a)=\bigoplus_{\gamma \in \mathcal{P}_{\sigma}} \mathbb{I}_{\mathcal{H}_{\gamma}} \otimes \pi_{\gamma}(a)
$$

with uniquely defined $\pi_{\gamma}(a) \in \mathcal{B}\left(\mathcal{K}_{\gamma}\right)$, for each $\gamma \in \mathcal{P}_{\sigma}$.

As $\pi$ is a *-representation, for each $\gamma \in \mathcal{P}_{\sigma}$, the map $\pi_{\gamma}: \mathcal{A}_{\Gamma_{0}}^{G} \ni a \mapsto \pi_{\gamma}(a) \in$ $\mathcal{B}\left(\mathcal{K}_{\gamma}\right)$ is a $*$-representation and we have

$$
\pi\left(\mathcal{A}_{\Gamma_{0}}^{G}\right)^{\prime \prime} \subset \bigoplus_{\gamma \in \mathcal{P}_{\sigma}} \mathbb{I}_{\mathcal{H}_{\gamma}} \otimes B\left(\mathcal{K}_{\gamma}\right)
$$

We claim that each $\pi_{\gamma}$ is an irreducible representation of $\mathcal{A}_{\Gamma_{0}}^{G}$, and (56) holds. To see this, note that for any $x \in \mathcal{B}\left(\mathcal{K}_{\gamma}\right)$, there exists a bounded net $\left\{a_{\lambda}\right\}_{\lambda} \in \mathcal{A}$ such that $\pi\left(a_{\lambda}\right)$ converges to $\mathbb{I}_{\mathcal{H}_{\gamma}} \otimes x \in \mathcal{B}\left(\mathcal{H}_{\gamma}\right) \otimes \mathcal{B}\left(\mathcal{K}_{\gamma}\right) \subset \mathcal{B}(\mathcal{H})$ in the $\sigma$-strong topology, by the irreducibility of $\pi$ and the Kaplansky density theorem. For this $\left\{a_{\lambda}\right\}_{\lambda}$, we have

$$
\begin{aligned}
\frac{1}{|G|} \sum_{g \in G} u_{g} \pi\left(a_{\lambda}\right) u_{g}^{*} & =\pi\left(\frac{1}{|G|} \sum_{g \in G} \tau_{\Gamma_{0}}(g)\left(a_{\lambda}\right)\right) \\
& =\bigoplus_{\gamma \in \mathcal{P}_{\sigma}} \mathbb{I}_{\mathcal{H}_{\gamma}} \otimes \pi_{\gamma}\left(\frac{1}{|G|} \sum_{g \in G} \tau_{\Gamma_{0}}(g)\left(a_{\lambda}\right)\right) \in \pi\left(\mathcal{A}_{\Gamma_{0}}^{G}\right)^{\prime \prime}
\end{aligned}
$$

because $\frac{1}{|G|} \sum_{g \in G} \tau_{\Gamma_{0}}(g)\left(a_{\lambda}\right)$ is $\tau_{\Gamma_{0}}$-invariant. Since the left hand side of (59) converges to $\mathbb{I}_{\mathcal{H}_{\gamma}} \otimes x$ in the $\sigma$-strong topology, we conclude that $\mathbb{I}_{\mathcal{H}_{\gamma}} \otimes x \in \pi\left(\mathcal{A}_{\Gamma_{0}}^{G}\right)^{\prime \prime}$. Hence (56) holds. Looking at the $\gamma$-component of (59), we see that $\pi_{\gamma}\left(\mathcal{A}_{\Gamma_{0}}^{G}\right)^{\prime \prime}=$ $\mathcal{B}\left(\mathcal{K}_{\gamma}\right)$. Hence $\pi_{\gamma}$ is irreducible. This completes the proof.

For each Lemma below, we use the machinery used in [FKK] and [KOS]. We remark arguments required to get a path inside of $\mathcal{A}_{\Gamma}^{G}$. 
Lemma 4.6. Let $\left(\mathcal{H}_{i}, \pi_{i}, u_{i}\right)$ with $i=0,1$ be irreducible covariant representations of $\Sigma_{\Gamma}^{(\sigma)}$ with irreducible decomposition of $u_{i}$ given by a set of Hilbert spaces $\left\{\mathcal{K}_{\gamma, i} \mid\right.$ $\left.\gamma \in \mathcal{P}_{\sigma}\right\}, i=0,1$. Let $\xi_{i} \in \mathcal{K}_{\alpha, i}, i=0,1$ be unit vectors. Let $\hat{\varphi}_{\xi_{i}}$ be a state on $\mathcal{B}\left(\mathcal{H}_{\alpha}\right) \otimes C^{*}\left(\Sigma_{\Gamma}^{(\sigma)}\right)$ given by $\left(\mathcal{H}_{i}, \pi_{i}, u_{i}, \xi_{i}\right)$, for each $i=0,1$. (Recall Notation 4.1 .) Then for any $\mathcal{F} \Subset \mathcal{B}\left(\mathcal{H}_{\alpha}\right) \otimes C^{*}\left(\Sigma_{\Gamma}^{(\sigma)}\right)$ and any $\varepsilon>0$, there exists a self-adjoint $h \in \mathcal{A}_{\Gamma}^{G}$ such that

$$
\left|\hat{\varphi}_{\xi_{0}}(x)-\hat{\varphi}_{\xi_{1}} \circ \operatorname{Ad}\left(e^{i h}\right)(x)\right|<\varepsilon, \quad x \in \mathcal{F} .
$$

Proof. First we prepare some notations. We denote by $\tilde{\xi}_{i}, \hat{\pi}_{i}$ the vector and the representation $\tilde{\xi}, \hat{\pi}$ defined in Notation 4.1 with $(\mathcal{H}, \pi, u, \xi)$ replaced by $\left(\mathcal{H}_{i}, \pi_{i}, u_{i}, \xi_{i}\right)$. (See (53) and (51).) The triple $\left(\mathcal{H}_{\alpha} \otimes \mathcal{H}_{i}, \hat{\pi}_{i}, \tilde{\xi}_{i}\right)$ is a GNS-triple of $\hat{\varphi}_{\xi_{i}}$. As $\mathcal{B}\left(\mathcal{H}_{\alpha}\right) \otimes C^{*}\left(\Sigma_{\Gamma}^{(\sigma)}\right)$ is simple, kernel of $\hat{\pi}_{i}$ is zero for each $i=0,1$. For each $k, j=1, \ldots, n_{\alpha}$, we define an element

$$
Q_{k, j}^{(\alpha)}:=\frac{n_{\alpha}}{|G|} \sum_{g \in G} \overline{\left\langle\psi_{k}^{(\alpha)}, V_{\alpha}(g) \psi_{j}^{(\alpha)}\right\rangle} \lambda_{g} \in C^{*}\left(\Sigma_{\Gamma}^{(\sigma)}\right),
$$

with $\lambda_{g} \in C^{*}\left(\Sigma_{\Gamma}^{(\sigma)}\right)$ introduced in section 3 (46). We also set

$$
R^{(\alpha)}:=\frac{1}{n_{\alpha}} \sum_{k, j=1}^{n_{\alpha}}\left|\psi_{k}^{(\alpha)}\right\rangle\left\langle\psi_{j}^{(\alpha)}\right| \otimes Q_{k, j}^{(\alpha)} \in \mathcal{B}\left(\mathcal{H}_{\alpha}\right) \otimes C^{*}\left(\Sigma_{\Gamma}^{(\sigma)}\right) .
$$

We claim

$$
\left(\pi_{i} \times u_{i}\right)\left(Q_{k, j}^{(\alpha)}\right)=\left|\psi_{k}^{(\alpha)}\right\rangle\left\langle\psi_{j}^{(\alpha)}\right| \otimes \mathbb{I}_{\mathcal{K}_{\alpha, i}} \in \mathcal{B}\left(\mathcal{H}_{\alpha} \otimes \mathcal{K}_{\alpha, i}\right) \subset \mathcal{B}\left(\mathcal{H}_{i}\right),
$$

for each $k, j=1, \ldots, n_{\alpha}$ and $i=0,1$. From this we have

$$
\hat{\pi}_{i}\left(R^{(\alpha)}\right)=\left|\Omega_{\alpha}\right\rangle\left\langle\Omega_{\alpha}\right| \otimes \mathbb{I}_{\mathcal{K}_{\alpha, i}}=: P^{(\alpha, i)}, \quad i=0,1 .
$$

From (64), we obtain

$$
\hat{\varphi}_{\xi_{i}}\left(R^{(\alpha)}\right)=1, \quad i=0,1 .
$$

To see (63), recall the orthogonality relation (8), the irreducible decomposition of $u_{i}$ given by $\left\{\mathcal{K}_{\gamma, i} \mid \gamma \in \mathcal{P}_{\sigma}\right\}$ and that $\left(\pi_{i} \times u_{i}\right)\left(\lambda_{g}\right)=u_{i}(g)$ (49). Then we have

$$
\begin{aligned}
\left(\pi_{i} \times u_{i}\right)\left(Q_{k, j}^{(\alpha)}\right) & =\frac{n_{\alpha}}{|G|} \sum_{g \in G} \overline{\left\langle\psi_{k}^{(\alpha)}, V_{\alpha}(g) \psi_{j}^{(\alpha)}\right\rangle} u_{i}(g) \\
& =\frac{n_{\alpha}}{|G|} \sum_{g \in G} \overline{\left\langle\psi_{k}^{(\alpha)}, V_{\alpha}(g) \psi_{j}^{(\alpha)}\right\rangle}\left(\bigoplus_{\gamma \in \mathcal{P}_{\sigma}} V_{\gamma}(g) \otimes \mathbb{I}_{\mathcal{K}_{\gamma, i}}\right) \\
& =\bigoplus_{\gamma \in \mathcal{P}_{\sigma}}\left(\delta_{\alpha, \gamma}\left|\psi_{k}^{(\alpha)}\right\rangle\left\langle\psi_{j}^{(\alpha)}\right| \otimes \mathbb{I}_{\mathcal{K}_{\alpha, i}}\right) .
\end{aligned}
$$

We now start the proof of Lemma. We fix an arbitrary $\mathcal{F} \Subset \mathcal{B}\left(\mathcal{H}_{\alpha}\right) \otimes C^{*}\left(\Sigma_{\Gamma}^{(\sigma)}\right)$ and $\varepsilon>0$. We then choose $0<\tilde{\varepsilon}$ small enough so that

$$
\tilde{\varepsilon}<\min \left\{1, \frac{\varepsilon}{2}\right\}, \quad \text { and } \quad 4 \max _{a \in \mathcal{F}}\|a\| \tilde{\varepsilon}^{\frac{1}{2}}<\frac{\varepsilon}{2} .
$$

We also set

$$
\tilde{\mathcal{F}}:=\mathcal{F} \cup\left\{R^{(\alpha)}\right\} \Subset B\left(\mathcal{H}^{(\alpha)}\right) \otimes C^{*}\left(\Sigma_{\Gamma}^{(\sigma)}\right) .
$$


Applying Lemma B.1 to this $\tilde{\varepsilon}$ and $\tilde{\mathcal{F}}$, and pure states $\hat{\varphi}_{\xi_{i}}, i=0,1$ of a simple unital $C^{*}$-algebra $B\left(\mathcal{H}^{(\alpha)}\right) \otimes C^{*}\left(\Sigma_{\Gamma}^{(\sigma)}\right)$, we obtain an $f \in\left(B\left(\mathcal{H}^{(\alpha)}\right) \otimes C^{*}\left(\Sigma_{\Gamma}^{(\sigma)}\right)\right)_{+, 1}$ and a unit vector $\zeta \in \mathcal{H}^{(\alpha)} \otimes \mathcal{H}_{1}$ such that

$$
\hat{\pi}_{1}(f) \zeta=\zeta, \quad\left\|f\left(a-\hat{\varphi}_{\xi_{0}}(a) \mathbb{I}\right) f\right\|<\tilde{\varepsilon}, \quad \text { for all } \quad a \in \tilde{\mathcal{F}} .
$$

For $P^{(\alpha, 1)}$ in (64) and the $\zeta$ in (69), we have

$$
\begin{aligned}
\left\|\left(\mathbb{I}-P^{(\alpha, 1)}\right) \zeta\right\|^{2} & =\left\langle\zeta, \hat{\pi}_{1}(f)\left(\mathbb{I}-\hat{\pi}_{1}\left(R^{(\alpha)}\right)\right) \hat{\pi}_{1}(f) \zeta\right\rangle \\
& =\left\langle\zeta, \hat{\pi}_{1}(f)\left(\hat{\varphi}_{\xi_{0}}\left(R^{(\alpha)}\right) \mathbb{I}-\hat{\pi}_{1}\left(R^{(\alpha)}\right)\right) \hat{\pi}_{1}(f) \zeta\right\rangle \\
& \leq\left\|f\left(\hat{\varphi}_{\xi_{0}}\left(R^{(\alpha)}\right) \mathbb{I}-R^{(\alpha)}\right) f\right\|<\tilde{\varepsilon} .
\end{aligned}
$$

Here we used (65), for the second equality. For the inequality we used (69) and $R^{(\alpha)} \in \tilde{\mathcal{F}}(68)$. Therefore, $P^{(\alpha, 1)} \zeta$ is not zero, and we may define a unit vector

$$
\tilde{\zeta}:=\frac{1}{\left\|P^{(\alpha, 1)} \zeta\right\|} P^{(\alpha, 1)} \zeta \in \mathcal{H}_{\alpha} \otimes \mathcal{H}_{1} .
$$

Furthermore, it satisfies

$$
\|\zeta-\tilde{\zeta}\| \leq 2 \tilde{\varepsilon}^{\frac{1}{2}}
$$

From this and two properties in (69) for any $a \in \mathcal{F}$, we have

$$
\begin{aligned}
& \left|\hat{\varphi}_{\xi_{0}}(a)-\left\langle\tilde{\zeta}, \hat{\pi}_{1}(a) \tilde{\zeta}\right\rangle\right| \leq\left|\left\langle\hat{\pi}_{1}(f) \zeta,\left(\hat{\varphi}_{\xi_{0}}(a)-\hat{\pi}_{1}(a)\right) \hat{\pi}_{1}(f) \zeta\right\rangle\right| \\
& \quad+\left|\left\langle\zeta, \hat{\pi}_{1}(a) \zeta\right\rangle-\left\langle\tilde{\zeta}, \hat{\pi}_{1}(a) \tilde{\zeta}\right\rangle\right| \\
& \quad \leq\left\|f\left(a-\hat{\varphi}_{\xi_{0}}(a) \mathbb{I}\right) f\right\|+2 \max _{a \in \mathcal{F}}\|a\|\|\zeta-\tilde{\zeta}\|<\tilde{\varepsilon}+2 \max _{a \in \mathcal{F}}\|a\| 2 \tilde{\varepsilon}^{\frac{1}{2}}<\varepsilon
\end{aligned}
$$

by the choice of $\tilde{\varepsilon}(67)$.

Since $P^{(\alpha, 1)} \tilde{\zeta}=\tilde{\zeta}$, there exists a unit vector $\eta \in \mathcal{K}_{\alpha, 1}$ such that $\tilde{\zeta}=\Omega_{\alpha} \otimes \eta$. By Lemma 4.4, for each $\gamma \in \mathcal{P}_{\sigma}$, there exists an irreducible representation $\pi_{\gamma, 1}$ of $\mathcal{A}_{\Gamma}^{G}$ on $\mathcal{K}_{\gamma, 1}$ such that

$$
\pi_{1}(a)=\bigoplus_{\gamma \in \mathcal{P}_{\sigma}} \mathbb{I}_{\mathcal{H}_{\gamma}} \otimes \pi_{\gamma, 1}(a), \quad a \in \mathcal{A}_{\Gamma}^{G}
$$

Applying the Kadison transitivity theorem for unit vectors $\xi_{1}, \eta \in \mathcal{K}_{\alpha, 1}$ and an irreducible representation $\left(\mathcal{K}_{\alpha, 1}, \pi_{\alpha, 1}\right)$ of $\mathcal{A}_{\Gamma}^{G}$, we obtain a self-adjoint $h \in \mathcal{A}_{\Gamma}^{G}$ such that $\pi_{\alpha, 1}\left(e^{-i h}\right) \xi_{1}=\eta$. With this $h$, we can write $\tilde{\zeta}$ as

$$
\tilde{\zeta}=\hat{\pi}_{1}\left(\mathbb{I}_{B\left(\mathcal{H}_{\alpha}\right)} \otimes e^{-i h}\right) \tilde{\xi}_{1} .
$$

Hence we obtain

$$
\hat{\varphi}_{\xi_{1}} \circ \operatorname{Ad}\left(e^{i h}\right)=\left\langle\tilde{\zeta}, \hat{\pi}_{1}(\cdot) \tilde{\zeta}\right\rangle .
$$

Combining this with (73), we see that (60) holds.

Remark 4.7. The main difference of the proof of Lemma 4.6 from [KOS], [FKK] is that in order to find $h$ in $\mathcal{A}_{\Gamma}^{G}$, we add $R^{(\alpha)}$ to $\mathcal{F}$. This allows us to replace $\zeta$ with $\tilde{\zeta}=\Omega_{\alpha} \otimes \eta$. From this combined with Lemma 4.4 the problem is reduced to the Kadison transitivity for the irreducible $\left(\mathcal{K}_{\alpha, 1}, \pi_{\alpha, 1}\left(\mathcal{A}_{\Gamma}^{G}\right)\right)$. Note that $R^{(\alpha)}$ belongs 
to $B\left(\mathcal{H}_{\alpha}\right) \otimes C^{*}\left(\Sigma_{\Gamma}^{(\sigma)}\right)$ but not in $\mathcal{A}_{\Gamma}$. By extending the $C^{*}$-algebra we consider, we are allowed to have the projection $P^{(\alpha, i)}$ (64) corresponding to the irreducible component of $u_{i}$ in the $C^{*}$-algebra.

Notation 4.8. For $\Lambda \Subset \Gamma$, we introduce a finite subset of $B\left(\mathcal{H}_{\alpha}\right) \otimes C^{*}\left(\Sigma_{\Gamma}^{(\sigma)}\right)$ given by

$$
\mathcal{G}_{\Lambda}:=\left\{\frac{1}{\sqrt{|G|}}\left|\psi_{j}^{(\alpha)}\right\rangle\left\langle\psi_{1}^{(\alpha)}\left|\otimes \lambda_{g} E_{I, I_{0}}^{(\Lambda)} \quad\right| \quad j=1, \ldots, n_{\alpha}, I \in\{1, \ldots, d\}^{\times \Lambda}, g \in G\right\} .\right.
$$

Here, we set $I_{0}:=\left(i_{k}\right)_{k \in \Lambda} \in\{1, \ldots, d\}^{\times \Lambda}$, with $i_{k}=1$ for all $k \in \Lambda$.

Notation 4.9. We say an irreducible covariant representation $(\mathcal{H}, \pi, u)$ of $\Sigma_{\Gamma}^{(\sigma)}$ and unit vectors $\xi, \eta \in \mathcal{H}_{\alpha} \otimes \mathcal{H}$ satisfy Condition 1 for a pair $\delta>0, \Lambda \Subset \Gamma$, if the representation $\hat{\pi}:=\operatorname{id}_{\mathcal{B}\left(\mathcal{H}_{\alpha}\right)} \otimes(\pi \times u)$ of $B\left(\mathcal{H}_{\alpha}\right) \otimes C^{*}\left(\Sigma_{\Gamma}^{(\sigma)}\right)$ satisfies the following:

(1) For any $x, y \in \mathcal{G}_{\Lambda}, \hat{\pi}(x)^{*} \xi$ and $\hat{\pi}(y)^{*} \eta$ are orthogonal.

(2) For any $x, y \in \mathcal{G}_{\Lambda}$,

$$
\left|\left\langle\xi, \hat{\pi}\left(x y^{*}\right) \xi\right\rangle-\left\langle\eta, \hat{\pi}\left(x y^{*}\right) \eta\right\rangle\right|<\delta .
$$

Let $\delta_{2, a}$ be the function given in Lemma B.4

Lemma 4.10. For any $\varepsilon>0$ and $\Lambda \Subset \Gamma$, there exists a $\delta_{1}(\varepsilon, \Lambda)>0$ satisfying the following: For any irreducible covariant representation $(\mathcal{H}, \pi, u)$ of $\Sigma_{\Gamma}^{(\sigma)}$ and unit vectors $\xi, \eta \in \mathcal{H}_{\alpha} \otimes \mathcal{H}$ satisfying Condition 1 for a pair $\delta_{1}(\varepsilon, \Lambda)>0, \Lambda \Subset \Gamma$, there exists a positive element $h$ of $\left(\mathcal{A}_{\Gamma \backslash \Lambda}^{G}\right)_{1}$ such that

$$
\left\|e^{i \pi \hat{\pi}(h)} \xi-\eta\right\|<\frac{1}{4 \sqrt{2}} \delta_{2, a}\left(\frac{\varepsilon}{8}\right) .
$$

Proof. Recall Lemma B.6. We set

$$
\delta_{1}(\varepsilon, \Lambda):=\delta_{3, a}\left(\varepsilon, n_{\alpha} d^{|\Lambda|}|G|\right),
$$

with $\delta_{3, a}(\cdot, \cdot)$ in Lemma B.6. We prove that this $\delta_{1}$ satisfies the condition above.

Let us consider an arbitrary irreducible covariant representation $(\mathcal{H}, \pi, u)$ of $\Sigma_{\Gamma}^{(\sigma)}$ and unit vectors $\xi, \eta \in \mathcal{H}_{\alpha} \otimes \mathcal{H}$ satisfying Condition 1 for a pair $\delta_{1}(\varepsilon, \Lambda)>0, \Lambda \Subset \Gamma$. We again use the notation $\hat{\pi}$ (51) for this $\pi$.

We apply Lemma B.6, to an infinite dimensional Hilbert space $\mathcal{H}_{\alpha} \otimes \mathcal{H}$, a unital $C^{*}$-algebra $\hat{\pi}\left(\mathcal{B}\left(\mathcal{H}_{\alpha}\right) \otimes C^{*}\left(\Sigma_{\Gamma}^{(\sigma)}\right)\right)$ acting irreducibly on $\mathcal{H}_{\alpha} \otimes \mathcal{H}$, a finite subset $\hat{\pi}\left(\mathcal{G}_{\Lambda}\right)$ of $\hat{\pi}\left(\mathcal{B}\left(\mathcal{H}_{\alpha}\right) \otimes C^{*}\left(\Sigma_{\Gamma}^{(\sigma)}\right)\right)$, and unit vectors $\xi, \eta \in \mathcal{H}_{\alpha} \otimes \mathcal{H}$. Note that $\sum_{x \in \hat{\pi}\left(\mathcal{G}_{\Lambda}\right)} x x^{*}=\mathbb{I}$ by the definition of $\mathcal{G}_{\Lambda}$. From Condition $1, \xi, \eta$ satisfy the required conditions in Lemma B.6. By Lemma B.6, there exists a positive $\tilde{h} \in\left(\mathcal{B}\left(\mathcal{H}_{\alpha}\right) \otimes C^{*}\left(\Sigma_{\Gamma}^{(\sigma)}\right)\right)_{+, 1}$ such that

$$
\begin{gathered}
\|\hat{\pi}(\bar{h})(\xi+\eta)\|<\frac{1}{4 \sqrt{2}} \delta_{2, a}\left(\frac{\varepsilon}{8}\right) e^{-\pi}, \quad \text { and } \\
\|(\mathbb{I}-\hat{\pi}(\bar{h}))(\xi-\eta)\|<\frac{1}{4 \sqrt{2}} \delta_{2, a}\left(\frac{\varepsilon}{8}\right) e^{-\pi},
\end{gathered}
$$


for

$$
\bar{h}:=\sum_{x \in \mathcal{G}_{\Lambda}} x \tilde{h} x^{*}
$$

Here the function $\delta_{2, a}$ is given in Theorem B.4. By this definition of $\bar{h}$, we see that

$$
\bar{h} \in\left(\mathcal{B}\left(\mathcal{H}_{\alpha}\right) \otimes \mathcal{A}_{\Lambda}\right)^{\prime} \cap\left\{\lambda_{g} \mid g \in G\right\}^{\prime} \cap\left(\mathcal{B}\left(\mathcal{H}_{\alpha}\right) \otimes C^{*}\left(\Sigma_{\Gamma}^{(\sigma)}\right)\right)_{+, 1} .
$$

We would like to replace $\bar{h}$ in (81) to some positive element $h \in\left(\mathcal{A}_{\Gamma \backslash \Lambda}^{G}\right)_{+, 1}$. In order to do so, we factorize $(\mathcal{H}, \pi, u)$ to $\Lambda$-part and $\Gamma \backslash \Lambda$-part: As in the proof of Theorem 2.4 there exists an irreducible covariant representation $(\tilde{\mathcal{H}}, \tilde{\pi}, \tilde{u})$ of $\Sigma_{\Gamma \backslash \Lambda}^{(\sigma)}$ and a unitary $W: \mathcal{H} \rightarrow\left(\otimes_{\Lambda} \mathbb{C}^{d}\right) \otimes \tilde{\mathcal{H}}$ such that

$$
W \pi(a) W^{*}=\left(\operatorname{id}_{\mathcal{A}_{\Lambda}} \otimes \tilde{\pi}\right)(a), \quad a \in \mathcal{A}_{\Gamma}
$$

and

$$
W u(g) W^{*}=\left(\bigotimes_{\Lambda} U(g)\right) \otimes \tilde{u}(g), \quad g \in G .
$$

By Lemma 1.1, $\tilde{u}$ has an irreducible decomposition of given by a set of Hilbert spaces $\left\{\mathcal{K}_{\gamma} \mid \gamma \in \mathcal{P}_{\sigma}\right\}$. By Lemma 4.4 and Lemma 1.1 we have

$$
\tilde{\pi}\left(\mathcal{A}_{\Gamma \backslash \Lambda}^{G}\right)^{\prime \prime}=\bigoplus_{\gamma \in \mathcal{P}_{\sigma}} \mathbb{I}_{\mathcal{H}_{\gamma}} \otimes B\left(\mathcal{K}_{\gamma}\right)=\tilde{u}(G)^{\prime} .
$$

Recall (81). Choose $\delta>0$ so that

$$
\begin{gathered}
\|\hat{\pi}(\bar{h})(\xi+\eta)\|+\delta<\frac{1}{4 \sqrt{2}} \delta_{2, a}\left(\frac{\varepsilon}{8}\right) e^{-\pi}, \quad \text { and } \\
\|(\mathbb{I}-\hat{\pi}(\bar{h}))(\xi-\eta)\|+\delta<\frac{1}{4 \sqrt{2}} \delta_{2, a}\left(\frac{\varepsilon}{8}\right) e^{-\pi} .
\end{gathered}
$$

As $\bar{h}$ is in $\left(\mathcal{B}\left(\mathcal{H}_{\alpha}\right) \otimes \mathcal{A}_{\Lambda}\right)^{\prime}$, from (84), we see that there exists a positive $y \in \mathcal{B}(\tilde{\mathcal{H}})_{1}$ such that

$$
\left(\mathbb{I}_{\mathcal{H}_{\alpha}} \otimes W\right) \hat{\pi}(\bar{h})\left(\mathbb{I}_{\mathcal{H}_{\alpha}} \otimes W^{*}\right)=\mathbb{I}_{\mathcal{H}_{\alpha}} \otimes \mathbb{I}_{\bigotimes_{\Lambda} \mathbb{C}^{d}} \otimes y .
$$

Furthermore, as $\bar{h}$ is in $\left\{\lambda_{g} \mid g \in G\right\}^{\prime}$, from (85), $y$ belongs to $\tilde{u}(G)^{\prime}=\tilde{\pi}\left(\mathcal{A}_{\Gamma \backslash \Lambda}^{G}\right)^{\prime \prime}$ by (86). By the Kaplansky density theorem, there exists a positive $h \in\left(\mathcal{A}_{\Gamma \backslash \Lambda}^{G}\right)_{+, 1}$ such that

$$
\|(\hat{\pi}(h)-\hat{\pi}(\bar{h}))(\xi \pm \eta)\|=\left\|\left(\mathbb{I}_{\mathcal{H}_{\alpha}} \otimes \mathbb{I}_{\bigotimes_{\Lambda} \mathbb{C}^{d}} \otimes(\tilde{\pi}(h)-y)\right)\left(\mathbb{I}_{\mathcal{H}_{\alpha}} \otimes W\right)(\xi \pm \eta)\right\|<\delta
$$

This $h$ satisfies

$$
\|\hat{\pi}(h)(\xi+\eta)\| \leq\|(\hat{\pi}(h)-\hat{\pi}(\bar{h}))(\xi+\eta)\|+\|\hat{\pi}(\bar{h})(\xi+\eta)\|<\frac{1}{4 \sqrt{2}} \delta_{2, a}\left(\frac{\varepsilon}{8}\right) e^{-\pi},
$$

and

$$
\begin{aligned}
&\|(\mathbb{I}-\hat{\pi}(h))(\xi-\eta)\| \leq\|(\hat{\pi}(h)-\hat{\pi}(\bar{h}))(\xi-\eta)\|+\|(\mathbb{I}-\hat{\pi}(\bar{h}))(\xi-\eta)\| \\
&<\frac{1}{4 \sqrt{2}} \delta_{2, a}\left(\frac{\varepsilon}{8}\right) e^{-\pi},
\end{aligned}
$$


from the choice of $\delta$, (87). We then obtain the required property of $h$ :

$$
\begin{aligned}
& \left\|e^{i \pi \hat{\pi}(h)} \xi-\eta\right\| \leq\left\|\frac{1}{2}\left(e^{i \pi \hat{\pi}(h)}(\xi+\eta)-(\xi+\eta)\right)\right\| \\
& \quad+\left\|\frac{1}{2}\left(e^{i \pi \hat{\pi}(h)}(\xi-\eta)+(\xi-\eta)\right)\right\| \\
& \quad \leq \frac{e^{\pi}}{2}\|\hat{\pi}(h)(\xi+\eta)\|+\frac{e^{\pi}}{2}\|(\mathbb{I}-\hat{\pi}(h))(\xi-\eta)\|<\frac{1}{4 \sqrt{2}} \delta_{2, a}\left(\frac{\varepsilon}{8}\right) .
\end{aligned}
$$

Remark 4.11. Note that an average over $G$ is contained in (82). Because of this, we could take $\bar{h}$ to be Ad $\lambda_{g}$-invariant. This is possible because $\lambda_{g}$ is included in the $C^{*}$-algebra we consider, i.e., in $\mathcal{B}\left(\mathcal{H}_{\alpha}\right) \otimes C^{*}\left(\Sigma_{\Gamma}^{(\sigma)}\right)$.

The main difference of Lemma 4.10 compared to [KOS] is replacing $\bar{h}$ with $h \in$ $\mathcal{A}_{\Gamma \backslash \Lambda}^{G}$. To carry it out, the decomposition (86) given from Lemma 1.1 Lemma 4.4 is used. This decomposition reduces the problem to the Kaplansky density Theorem for $\hat{\pi}\left(\mathcal{A}_{\Gamma \backslash \Lambda}^{G}\right)$.

Notation 4.12. For any $\varepsilon>0$ and a finite set $\mathcal{F} \Subset \mathcal{A}$, there exists a $\Lambda(\varepsilon, \mathcal{F}) \Subset \Gamma$ such that

$$
\inf \left\{\|a-b\| \mid b \in \mathcal{A}_{\Lambda(\varepsilon, \mathcal{F})}\right\}<\frac{\varepsilon}{16}, \quad \text { for all } \quad a \in \mathcal{F} .
$$

For each $\varepsilon>0$ and $\mathcal{F} \Subset \mathcal{A}$, we fix such $\Lambda(\varepsilon, \mathcal{F})$. If $\mathcal{F}$ is included in $\mathcal{A}_{\Lambda}$ for some $\Lambda \Subset \Gamma$, we choose $\Lambda(\varepsilon, \mathcal{F})$ so that $\Lambda(\varepsilon, \mathcal{F}) \subset \Lambda$. For any $\varepsilon>0$ and $\mathcal{F} \Subset \mathcal{A}$, set

$$
\delta_{2}(\varepsilon, \mathcal{F}):=\frac{1}{2} \delta_{1}\left(\frac{\varepsilon}{4}, \Lambda(\varepsilon, \mathcal{F})\right) .
$$

Here we used the function $\delta_{1}$ introduced in Lemma 4.10.

Lemma 4.13. Let $\varepsilon>0$, and $\mathcal{F} \Subset\left(\mathcal{A}_{\Gamma}\right)_{1}$. Let $(\mathcal{H}, \pi, u)$ be an irreducible covariant representation of $\Sigma_{\Gamma}^{(\sigma)}$ with an irreducible decomposition of u given by a set of Hilbert spaces $\left\{\mathcal{K}_{\gamma} \mid \gamma \in \mathcal{P}_{\sigma}\right\}$. Let $\xi, \eta$ be unit vectors in $\mathcal{K}_{\alpha}$. Suppose that unit vectors

$$
\tilde{\xi}:=\Omega_{\alpha} \otimes \xi, \quad \tilde{\eta}:=\Omega_{\alpha} \otimes \eta \in \mathcal{H}_{\alpha} \otimes \mathcal{H}
$$

satisfy

$$
\left|\left\langle\tilde{\eta}, \hat{\pi}\left(x y^{*}\right) \tilde{\eta}\right\rangle-\left\langle\tilde{\xi}, \hat{\pi}\left(x y^{*}\right) \tilde{\xi}\right\rangle\right|<\delta_{2}(\varepsilon, \mathcal{F}), \quad \text { for all } \quad x, y \in \mathcal{G}_{\Lambda(\varepsilon, \mathcal{F})} .
$$

(Recall Notation 4.1 and Notation 4.8.) Then there exists a norm-continuous path of unitaries $v:[0,1] \rightarrow \mathcal{U}\left(\mathcal{A}_{\Gamma}^{G}\right)$ such that $v(0)=\mathbb{I}_{\mathcal{A}_{\Gamma}}$,

$$
\tilde{\eta}=\left(\mathbb{I}_{\mathcal{H}_{\alpha}} \otimes \pi(v(1))\right) \tilde{\xi},
$$

and

$$
\sup _{t \in[0,1]}\|\operatorname{Ad} v(t)(a)-a\|<\varepsilon, \quad \text { for all } \quad a \in \mathcal{F} .
$$

Proof. We denote by $\mathcal{N}$, the finite dimensional subspace spanned by $\left\{\pi\left(x y^{*}\right) \tilde{\xi}\right.$, $\left.\hat{\pi}\left(x y^{*}\right) \tilde{\eta} \mid x, y \in \mathcal{G}_{\Lambda(\varepsilon, \mathcal{F})}\right\}$. Then there exists a unit vector $\zeta$ in $\mathcal{N}^{\perp}$, the orthogonal complement of $\mathcal{N}$, such that

(98) $\left|\left\langle\zeta, \hat{\pi}\left(x y^{*}\right) \zeta\right\rangle-\left\langle\tilde{\xi}, \hat{\pi}\left(x y^{*}\right) \tilde{\xi}\right\rangle\right|<\delta_{2}(\varepsilon, \mathcal{F}) \leq \delta_{1}\left(\frac{\varepsilon}{4}, \Lambda(\varepsilon, \mathcal{F})\right), \quad x, y \in \mathcal{G}_{\Lambda(\varepsilon, \mathcal{F})}$. 
To see this, note that the intersection of the set of all compact operators on $\mathcal{H}_{\alpha} \otimes \mathcal{H}$ and $\hat{\pi}\left(\mathcal{B}\left(\mathcal{H}_{\alpha}\right) \otimes C^{*}\left(\Sigma_{\Gamma}^{(\sigma)}\right)\right)$ is 0 because $\mathcal{B}\left(\mathcal{H}_{\alpha}\right) \otimes C^{*}\left(\Sigma_{\Gamma}^{(\sigma)}\right)$ is simple. Applying Glimm's Lemma (Theorem B.5) to $\delta_{2}(\varepsilon, \mathcal{F})>0$, a pure state $\langle\tilde{\xi}, \tilde{\xi}\rangle$ on $\hat{\pi}\left(\mathcal{B}\left(\mathcal{H}_{\alpha}\right) \otimes C^{*}\left(\Sigma_{\Gamma}^{(\sigma)}\right)\right)$, a finite dimensional subspace $\mathcal{N}$ of $\mathcal{H}_{\alpha} \otimes \mathcal{H}$ and a finite subset $\hat{\pi}\left(\mathcal{G}_{\Lambda(\varepsilon, \mathcal{F})} \mathcal{G}_{\Lambda(\varepsilon, \mathcal{F})}^{*}\right)$, we obtain $\zeta$ above.

Combining (98) with (95) we also get

$$
\begin{aligned}
\left|\left\langle\zeta, \hat{\pi}\left(x y^{*}\right) \zeta\right\rangle-\left\langle\tilde{\eta}, \hat{\pi}\left(x y^{*}\right) \tilde{\eta}\right\rangle\right| \leq & \left|\left\langle\zeta, \hat{\pi}\left(x y^{*}\right) \zeta\right\rangle-\left\langle\tilde{\xi}, \hat{\pi}\left(x y^{*}\right) \tilde{\xi}\right\rangle\right| \\
& +\left|\left\langle\tilde{\eta}, \hat{\pi}\left(x y^{*}\right) \tilde{\eta}\right\rangle-\left\langle\tilde{\xi}, \hat{\pi}\left(x y^{*}\right) \tilde{\xi}\right\rangle\right| \\
< & 2 \delta_{2}(\varepsilon, \mathcal{F})=\delta_{1}\left(\frac{\varepsilon}{4}, \Lambda(\varepsilon, \mathcal{F})\right), \quad x, y \in \mathcal{G}_{\Lambda(\varepsilon, \mathcal{F})} .
\end{aligned}
$$

Hence $(\mathcal{H}, \pi, u)$ and unit vectors $\tilde{\xi}, \zeta$ (resp. $\tilde{\eta}, \zeta$ ) satisfy Condition 1 . (Notation 4.9) for a pair $\delta_{1}\left(\frac{\varepsilon}{4}, \Lambda(\varepsilon, \mathcal{F})\right)>0, \Lambda(\varepsilon, \mathcal{F}) \Subset \Gamma$. Therefore, from Lemma 4.10 there exist positive elements $h_{1}, h_{2}$ in $\left(\mathcal{A}_{\Gamma \backslash \Lambda(\varepsilon, \mathcal{F})}^{G}\right)_{1}$ such that

$$
\left\|e^{i \pi \hat{\pi}\left(h_{1}\right)} \tilde{\xi}-\zeta\right\|<\frac{1}{4 \sqrt{2}} \delta_{2, a}\left(\frac{\varepsilon}{32}\right), \quad \text { and } \quad\left\|e^{i \pi \hat{\pi}\left(h_{2}\right)} \tilde{\eta}-\zeta\right\|<\frac{1}{4 \sqrt{2}} \delta_{2, a}\left(\frac{\varepsilon}{32}\right) .
$$

Here $\delta_{2, a}$ is given in Theorem B.4. By the definition of $\tilde{\xi}$ (94) and the decomposition

$$
\pi(a)=\bigoplus_{\gamma \in \mathcal{P}_{\sigma}} \mathbb{I}_{\mathcal{H}_{\gamma}} \otimes \pi_{\gamma}(a), \quad a \in \mathcal{A}_{\Gamma}^{G}
$$

( (55) of Lemma 4.4), with irreducible *-representations $\left(\mathcal{K}_{\gamma}, \pi_{\gamma}\right)$ of $\mathcal{A}_{\Gamma}^{G}$, we have $e^{i \pi \hat{\pi}\left(h_{1}\right)} \tilde{\xi}=\Omega_{\alpha} \otimes e^{i \pi \pi_{\alpha}\left(h_{1)}\right.} \xi$. Similarly, we have $e^{i \pi \hat{\pi}\left(h_{2}\right)} \tilde{\eta}=\Omega_{\alpha} \otimes e^{i \pi \pi_{\alpha}\left(h_{2}\right)} \eta$. Combining this with (100), we see that the unit vectors $e^{i \pi \pi_{\alpha}\left(h_{1)}\right.} \xi, e^{i \pi \pi_{\alpha}\left(h_{2}\right)} \eta$ in $\mathcal{K}_{\alpha}$ satisfies

$$
\left\|e^{i \pi \pi_{\alpha}\left(h_{1}\right)} \xi-e^{i \pi \pi_{\alpha}\left(h_{2}\right)} \eta\right\|<\frac{1}{2 \sqrt{2}} \delta_{2, a}\left(\frac{\varepsilon}{32}\right) .
$$

Then from Lemma B.2, there exists a unitary $v_{0}$ on $\mathcal{K}_{\alpha}$ such that

$$
v_{0} e^{i \pi \pi_{\alpha}\left(h_{1}\right)} \xi=e^{i \pi \pi_{\alpha}\left(h_{2}\right)} \eta, \quad \text { and } \quad\left\|v_{0}-\mathbb{I}_{\mathcal{K}_{\alpha}}\right\|<\frac{1}{2} \delta_{2, a}\left(\frac{\varepsilon}{32}\right)<\delta_{2, a}\left(\frac{\varepsilon}{32}\right) .
$$

From this and the fact that $\pi_{\alpha}$ is an irreducible representation of $\mathcal{A}_{\Gamma}^{G}$, applying Theorem B.4, we obtain a self-adjoint $k \in \mathcal{A}_{\Gamma}^{G}$ such that

$$
e^{i \pi_{\alpha}(k)} e^{i \pi \pi_{\alpha}\left(h_{1}\right)} \xi=e^{i \pi \pi_{\alpha}\left(h_{2}\right)} \eta, \quad \text { and } \quad\|k\| \leq \delta_{1, a}\left(\frac{\varepsilon}{32}\right) .
$$

Here the function $\delta_{1, a}$ is given in Notation B.3.

Now we define a continuous path of unitaries $v:[0,1] \rightarrow \mathcal{U}\left(\mathcal{A}_{\Gamma}^{G}\right)$. Set

$$
\begin{aligned}
v_{1}(t) & :=e^{i t \pi h_{1}} \in \mathcal{U}\left(\mathcal{A}_{\Gamma \backslash \Lambda(\varepsilon, \mathcal{F})}^{G}\right), \\
v_{2}(t) & :=e^{i t k} \in \mathcal{U}\left(\mathcal{A}_{\Gamma}^{G}\right), \\
v_{3}(t) & :=e^{-i t \pi h_{2}} \in \mathcal{U}\left(\mathcal{A}_{\Gamma \backslash \Lambda(\varepsilon, \mathcal{F})}^{G}\right)
\end{aligned}
$$

for each $t \in[0,1]$. 
For $i=1,3$, as $v_{i}$ takes value in $\mathcal{U}\left(\mathcal{A}_{\Gamma \backslash \Lambda(\varepsilon, \mathcal{F})}^{G}\right), v_{i}(t)$ commutes with elements in $\mathcal{A}_{\Lambda(\varepsilon, \mathcal{F})}$. From this and the fact that the distance between $\mathcal{F}$ and $\mathcal{A}_{\Lambda(\varepsilon, \mathcal{F})}$ is less than $\frac{\varepsilon}{16}$ (Notation 4.12 (92)), we get $\left\|\operatorname{Ad} v_{i}(t)(a)-a\right\|<\frac{\varepsilon}{8}$, for all $a \in \mathcal{F}$, $t \in[0,1]$, and $i=1,3$. For $i=2$, from $\|k\| \leq \delta_{1, a}\left(\frac{\varepsilon}{32}\right)$, recalling the definition of $\delta_{1, a}$ in Notation B.3, we obtain $\left\|\operatorname{Ad} v_{2}(t)(a)-a\right\| \leq 2\left\|v_{2}(t)-\mathbb{I}\right\| \leq \frac{\varepsilon}{16}$, for all $a \in \mathcal{F} \subset\left(\mathcal{A}_{\Gamma}\right)_{1}$ and $t \in[0,1]$.

We define $v:[0,1] \rightarrow \mathcal{U}\left(\mathcal{A}_{\Gamma}^{G}\right)$ by

$$
v(t):=\left\{\begin{array}{c}
v_{1}(3 t), \quad t \in\left[0, \frac{1}{3}\right], \\
v_{2}\left(3\left(t-\frac{1}{3}\right)\right) v_{1}(1), \quad t \in\left[\frac{1}{3}, \frac{2}{3}\right], \\
v_{3}\left(3\left(t-\frac{2}{3}\right)\right) v_{2}(1) v_{1}(1), \quad t \in\left[\frac{2}{3}, 1\right] .
\end{array}\right.
$$

Clearly $v(0)=\mathbb{I}_{\mathcal{A}_{\Gamma}}$ and $v$ is norm-continuous, and it takes values in $\mathcal{U}\left(\mathcal{A}_{\Gamma}^{G}\right)$. From the above estimates on $\left\|\operatorname{Ad} v_{i}(t)(a)-a\right\|$ for $a \in \mathcal{F}$ and $i=1,2,3$, we also get (97). Furthermore, we have

$$
\left(\mathbb{I}_{\mathcal{H}_{\alpha}} \otimes \pi(v(1))\right) \tilde{\xi}=\Omega_{\alpha} \otimes \pi_{\alpha}(v(1)) \xi=\Omega_{\alpha} \otimes \pi_{\alpha}\left(e^{-i \pi h_{2}} e^{i k} e^{i \pi h_{1}}\right) \xi=\Omega_{\alpha} \otimes \eta=\tilde{\eta} .
$$

Here, for the first equality, we used the fact that $v(1)$ is in in $\mathcal{A}_{\Gamma}^{G}$ and (101). The third equality is from (104).

Remark 4.14. By Lemma4.10, we can take $h_{1}, h_{2}$ in the fixed point algebra $\mathcal{A}_{\Gamma \backslash \Lambda_{(\varepsilon, \mathcal{F})}}^{G}$. With the special form of $\tilde{\xi}, \tilde{\eta}$, in (94), the problem is reduced to the Kadison transitivity theorem for $\left(\mathcal{K}_{\alpha}, \pi_{\alpha}\left(\mathcal{A}_{\Gamma}^{G}\right)\right)$. The irreducibility of $\pi_{\alpha}$ is used there. From this we may obtain $k$ interpolating $e^{i \pi \hat{\pi}\left(h_{1}\right)} \tilde{\xi}$ and $e^{i \pi \hat{\pi}\left(h_{2}\right)} \tilde{\eta}$, from $\mathcal{A}_{\Gamma}^{G}$.

Lemma 4.15. For any $\varepsilon>0$ and $\mathcal{F} \Subset\left(\mathcal{A}_{\Gamma}\right)_{1}$, the following holds: Let $\left(\mathcal{H}_{i}, \pi_{i}, u_{i}\right)$ with $i=0,1$ be irreducible covariant representations of $\Sigma_{\Gamma}^{(\sigma)}$ with irreducible decomposition of $u_{i}$ given by a set of Hilbert spaces $\left\{\mathcal{K}_{\gamma, i} \mid \gamma \in \mathcal{P}_{\sigma}\right\}$. Let $\xi_{i} \in \mathcal{K}_{\alpha, i}$ be a unit vector in $\mathcal{K}_{\alpha, i}$ for $i=0,1$. Suppose that the representation $\hat{\pi}_{i}:=\operatorname{id}_{\mathcal{H}_{\alpha}} \otimes\left(\pi_{i} \times u_{i}\right)$, $i=0,1$ of $B\left(\mathcal{H}_{\alpha}\right) \otimes C^{*}\left(\Sigma_{\Gamma}^{(\sigma)}\right)$ and unit vectors $\tilde{\xi}_{i}:=\Omega_{\alpha} \otimes \xi_{i}$ in $\mathcal{H}_{\alpha} \otimes \mathcal{H}_{i}, i=0,1$ satisfy

$$
\left|\left\langle\tilde{\xi}_{0}, \hat{\pi}_{0}\left(x y^{*}\right) \tilde{\xi}_{0}\right\rangle-\left\langle\tilde{\xi}_{1}, \hat{\pi}_{1}\left(x y^{*}\right) \tilde{\xi}_{1}\right\rangle\right|<\frac{1}{2} \delta_{2}(\varepsilon, \mathcal{F}), \quad \text { for all } \quad x, y \in \mathcal{G}_{\Lambda(\varepsilon, \mathcal{F})} .
$$

(Recall Notation 4.12 for $\left.\delta_{2}.\right)$ Then for any $\varepsilon^{\prime}>0$ and $\mathcal{F}^{\prime} \Subset B\left(\mathcal{H}_{\alpha}\right) \otimes C^{*}\left(\Sigma_{\Gamma}^{(\sigma)}\right)$, there exists a norm-continuous path $v:[0,1] \rightarrow \mathcal{U}\left(\mathcal{A}_{\Gamma}^{G}\right)$ with $v(0)=\mathbb{I}_{\mathcal{A}_{\Gamma}}$ such that

$$
\left|\left\langle\tilde{\xi}_{0}, \hat{\pi}_{0}(a) \tilde{\xi}_{0}\right\rangle-\left\langle\tilde{\xi}_{1},\left(\hat{\pi}_{1} \circ \operatorname{Ad}(v(1))\right)(a) \tilde{\xi}_{1}\right\rangle\right|<\varepsilon^{\prime}, \quad \text { for all } \quad a \in \mathcal{F}^{\prime}
$$

and

$$
\|\operatorname{Ad} v(t)(y)-y\|<\varepsilon, \quad \text { for all } \quad y \in \mathcal{F}, \quad \text { and } \quad t \in[0,1] \text {. }
$$


Proof. From Lemma 4.6, there exists a self-adjoint $h \in \mathcal{A}_{\Gamma}^{G}$ such that

$$
\begin{aligned}
\mid\left\langle\tilde{\xi}_{0}, \hat{\pi}_{0}(a) \tilde{\xi}_{0}\right\rangle- & \left\langle\tilde{\xi}_{1}, \hat{\pi}_{1} \circ \operatorname{Ad}\left(e^{i h}\right)(a) \tilde{\xi}_{1}\right\rangle \mid \\
& <\min \left\{\varepsilon^{\prime}, \frac{1}{2} \delta_{2}(\varepsilon, \mathcal{F})\right\} \quad \text { for all } a \in \mathcal{F}^{\prime} \cup \mathcal{G}_{\Lambda(\varepsilon, \mathcal{F})}\left(\mathcal{G}_{\Lambda(\varepsilon, \mathcal{F})}\right)^{*} .
\end{aligned}
$$

From this and (108), we have

$\left|\left\langle\tilde{\xi}_{1}, \hat{\pi}_{1} \circ \operatorname{Ad}\left(e^{i h}\right)\left(x y^{*}\right) \tilde{\xi}_{1}\right\rangle-\left\langle\tilde{\xi}_{1}, \hat{\pi}_{1}\left(x y^{*}\right) \tilde{\xi}_{1}\right\rangle\right|<\delta_{2}(\varepsilon, \mathcal{F}) \quad$ for all $\quad x, y \in \mathcal{G}_{\Lambda(\varepsilon, \mathcal{F})}$.

Recall from Lemma 4.4 that

$$
\pi_{1}(a)=\bigoplus_{\gamma \in \mathcal{P}_{\sigma}} \mathbb{I}_{\mathcal{H}_{\gamma}} \otimes \pi_{\gamma, 1}(a), \quad a \in \mathcal{A}_{\Gamma}^{G}
$$

with irreducible $*$-representations $\left(\mathcal{K}_{\gamma, 1}, \pi_{\gamma, 1}\right)$ of $\mathcal{A}_{\Gamma}^{G}$. From this and $h \in \mathcal{A}_{\Gamma}^{G}$, we see that $\hat{\pi}_{1}\left(e^{-i h}\right) \tilde{\xi}_{1}=\Omega_{\alpha} \otimes \pi_{\alpha, 1}\left(e^{-i h}\right) \xi_{1}$ By (112) $,\left(\mathcal{H}_{1}, \pi_{1}, u_{1}\right), \xi_{1}, \pi_{\alpha, 1}\left(e^{-i h}\right) \xi_{1}$ satisfies the required condition in Lemma 4.13 .

Applying Lemma 4.13 for $\left(\mathcal{H}_{1}, \pi_{1}, u_{1}\right)$ and $\xi_{1}, \pi_{\alpha, 1}\left(e^{-i h}\right) \xi_{1}$, we obtain a normcontinuous path of unitaries $v:[0,1] \rightarrow \mathcal{U}\left(\mathcal{A}_{\Gamma}^{G}\right)$ such that $v(0)=\mathbb{I}_{\mathcal{A}_{\Gamma}}$,

$$
\hat{\pi}_{1}\left(e^{-i t h}\right) \tilde{\xi}_{1}=\hat{\pi}_{1}\left(v(1)^{*}\right) \tilde{\xi}_{1},
$$

and

$$
\sup _{t \in[0,1]}\|\operatorname{Ad} v(t)(a)-a\|<\varepsilon, \quad \text { for all } \quad a \in \mathcal{F} .
$$

From (111) and (114), we obtain (109).

Remark 4.16. As in [KOS, we replace $e^{i t h}$ with $v(t)$ which satisfy (115). We may do so with $v(t)$ in $\mathcal{A}_{\Gamma}^{G}$ because of Lemma 4.13

After these preparation, the proof of Proposition 4.2 is the same as proof of Theorem 2.1 of [KOS]. We give it here for the reader's convenience.

Proof of Proposition 4.2. We fix an increasing sequence $\Lambda_{n}, n=0,1,2, \ldots$ of nonempty finite subsets of $\Gamma$ such that $\Lambda_{n} \nearrow \Gamma$.

For each $i=0,1$, we use the notation $\hat{\pi}_{i}, \tilde{\xi}_{i}, \hat{\varphi}_{\xi_{i}}$ given in Notation 4.1 replacing $(\mathcal{H}, \pi, u)$ and $\xi \in \mathcal{K}_{\alpha}$ with $\left(\mathcal{H}_{i}, \pi_{i}, u_{i}\right)$ and $\xi_{i} \in \mathcal{K}_{\alpha, i}$. Let $\left(\mathcal{K}_{\alpha, i}, \pi_{\alpha, i}\right), i=0,1$ be the irreducible $*$-representation of $\mathcal{A}_{\Gamma}^{G}$ obtained in Lemma 4.4 (55) with $(\mathcal{H}, \pi, u)$, $\Gamma_{0}$ replaced by $\left(\mathcal{H}_{i}, \pi_{i}, u_{i}\right), \Gamma$.

Set $\mathcal{F}_{0}:=\mathcal{S}_{\Lambda_{0}}$. (Recall (11).) Fix $\varepsilon>0$ or set $\varepsilon=1$. Set $\mathcal{G}_{0}:=\mathcal{G}_{\Lambda\left(\varepsilon, \mathcal{F}_{0}\right)} \mathcal{G}_{\Lambda\left(\varepsilon, \mathcal{F}_{0}\right)}^{*}$. From Lemma 4.6, there exists a self-adjoint $h_{0} \in \mathcal{A}_{\Gamma}^{G}$. such that

$$
\left|\hat{\varphi}_{\xi_{0}} \circ \operatorname{Ad}\left(e^{i h_{0}}\right)(a)-\hat{\varphi}_{\xi_{1}}(a)\right|<\min \left\{\frac{1}{2} \delta_{2}\left(\varepsilon, \mathcal{F}_{0}\right), \varepsilon\right\}, \quad a \in \mathcal{G}_{0} \cup \mathcal{F}_{0} .
$$

(Recall Notation 4.12 for $\delta_{2}$.) We define $v_{0}:=[0,1] \rightarrow \mathcal{U}\left(\mathcal{A}_{\Gamma}^{G}\right)$ by

$$
v_{0}(t)=e^{i t h_{0}}, \quad t \in[0,1] .
$$


We consider the following proposition $\left[P_{n}\right]$ for each $n \in \mathbb{N}$ :

$\left[P_{n}\right]$ There exist norm-continuous paths $v_{k}:[0,1] \rightarrow \mathcal{U}\left(\mathcal{A}_{\Gamma}^{G}\right), k=$ $0, \ldots, 2 n$ with $v_{k}(0)=\mathbb{I}_{\mathcal{A}_{\Gamma}}$ satisfying the following: Set

$$
\begin{aligned}
\mathcal{F}_{2 j}:= & \left\{x, \operatorname{Ad}\left(v_{2 j-1}(1)^{*} v_{2 j-3}(1)^{*} \cdots v_{3}(1)^{*} v_{1}(1)^{*}\right)(x) \mid x \in \mathcal{S}_{\Lambda_{2 j}}\right\}, \quad j=1, \ldots, n, \\
& \text { and } \\
(119) \quad & \mathcal{F}_{2 j-1}:=\left\{x, \operatorname{Ad}\left(v_{2 j-2}(1)^{*} v_{2 j-4}(1)^{*}\right.\right. \\
& \left.\left.\quad \cdots v_{4}(1)^{*} v_{2}(1)^{*} v_{0}(1)^{*}\right)(x) \mid x \in \mathcal{S}_{\Lambda_{2 j-1}}\right\}, \quad j=1, \ldots, n .
\end{aligned}
$$

(Recall (11).) We also denote the finite subset $\mathcal{G}_{\Lambda\left(\frac{\varepsilon}{2^{k}}, \mathcal{F}_{k}\right)} \mathcal{G}_{\Lambda\left(\frac{\varepsilon}{2^{k}}, \mathcal{F}_{k}\right)}$ by $\mathcal{G}_{k}$, for each $k=0,1, \ldots, 2 n$. Then the following three inequalities hold.

(1) For all $a \in \mathcal{G}_{2 n} \cup \mathcal{F}_{2 n}$,

$$
\left|\hat{\varphi}_{\xi_{0}} \circ \operatorname{Ad}\left(v_{0}(1) v_{2}(1) \cdots v_{2 n}(1)\right)(a)-\hat{\varphi}_{\xi_{1}} \circ \operatorname{Ad}\left(v_{1}(1) v_{3}(1) \cdots v_{2 n-1}(1)\right)(a)\right|
$$

$<\min \left\{\frac{1}{2} \delta_{2}\left(\frac{\varepsilon}{2^{2 n}}, \mathcal{F}_{2 n}\right), \frac{\varepsilon}{2^{2 n}}\right\}$

(2) For all $a \in \mathcal{G}_{2 n-1} \cup \mathcal{F}_{2 n-1}$,

$$
\begin{aligned}
\left|\hat{\varphi}_{\xi_{0}} \circ \operatorname{Ad}\left(v_{0}(1) v_{2}(1) \cdots v_{2 n-2}(1)\right)(a)-\hat{\varphi}_{\xi_{1}} \circ \operatorname{Ad}\left(v_{1}(1) v_{3}(1) \cdots v_{2 n-1}(1)\right)(a)\right| \\
\quad<\min \left\{\frac{1}{2} \delta_{2}\left(\frac{\varepsilon}{2^{2 n-1}}, \mathcal{F}_{2 n-1}\right), \frac{\varepsilon}{2^{2 n-1}}\right\}
\end{aligned}
$$

(3) For all $t \in[0,1], k=1,2, \ldots, 2 n$ with $k \leq 2 n$ and $x \in \mathcal{F}_{k-1}$, we have

$$
\left\|\operatorname{Ad} v_{k}(t)(x)-x\right\|<\frac{\varepsilon}{2^{k-1}} .
$$

Let us check that $\left[P_{1}\right]$ with $v_{0}$ given in (117) holds. Set $\mathcal{F}_{1}$ as in (119) with $j=1$ and this $v_{0}$. Set $\mathcal{G}_{1}:=\mathcal{G}_{\Lambda\left(\frac{\varepsilon}{2}, \mathcal{F}_{1}\right)} \mathcal{G}_{\Lambda\left(\frac{\varepsilon}{2}, \mathcal{F}_{1}\right)}^{*}$. From (116), applying Lemma 4.15 for vectors $\xi_{1}$ and $\pi_{\alpha, 0}\left(v_{0}(1)^{*}\right) \xi_{0}$, there exists a norm-continuous path $v_{1}:[0,1] \rightarrow \mathcal{U}\left(\mathcal{A}_{\Gamma}^{G}\right)$ with $v_{1}(0)=\mathbb{I}$ such that

$$
\begin{aligned}
\left|\hat{\varphi}_{\xi_{0}} \circ \operatorname{Ad}\left(v_{0}(1)\right)(a)-\hat{\varphi}_{\xi_{1}} \circ \operatorname{Ad}\left(v_{1}(1)\right)(a)\right| \\
\quad<\min \left\{\frac{1}{2} \delta_{2}\left(\frac{\varepsilon}{2}, \mathcal{F}_{1}\right), \frac{\varepsilon}{2}\right\}, \quad \text { for all } \quad a \in \mathcal{G}_{1} \cup \mathcal{F}_{1}
\end{aligned}
$$

and

$$
\left\|\operatorname{Ad} v_{1}(t)(y)-y\right\|<\varepsilon, \quad \text { for all } \quad y \in \mathcal{F}_{0}, \quad \text { and } \quad t \in[0,1] .
$$

Set $\mathcal{F}_{2}$ as in (118) with $j=1$ for this $v_{1}$. And set $\mathcal{G}_{2}:=\mathcal{G}_{\Lambda\left(\frac{\varepsilon}{2^{2}}, \mathcal{F}_{2}\right)} \mathcal{G}_{\Lambda\left(\frac{\varepsilon}{2^{2}}, \mathcal{F}_{2}\right)}$. From (123), applying Lemma 4.15] again to vectors $\pi_{\alpha, 0}\left(v_{0}(1)^{*}\right) \xi_{0}$ and $\pi_{\alpha, 1}\left(v_{1}(1)^{*}\right) \xi_{1}$, we obtain a norm-continuous path $v_{2}:[0,1] \rightarrow \mathcal{U}\left(\mathcal{A}_{\Gamma}^{G}\right)$ with $v_{2}(0)=\mathbb{I}$ such that

$$
\begin{aligned}
\left|\hat{\varphi}_{\xi_{0}} \circ \operatorname{Ad}\left(v_{0}(1) v_{2}(1)\right)(a)-\hat{\varphi}_{\xi_{1}} \circ \operatorname{Ad}\left(v_{1}(1)\right)(a)\right| \\
\quad<\min \left\{\frac{1}{2} \delta_{2}\left(\frac{\varepsilon}{2^{2}}, \mathcal{F}_{2}\right), \frac{\varepsilon}{2^{2}}\right\}, \quad \text { for all } \quad a \in \mathcal{G}_{2} \cup \mathcal{F}_{2}
\end{aligned}
$$


and

$$
\left\|\operatorname{Ad} v_{2}(t)(y)-y\right\|<\frac{\varepsilon}{2}, \quad \text { for all } \quad y \in \mathcal{F}_{1}, \quad \text { and } \quad t \in[0,1] .
$$

Hence we have proven $\left[P_{1}\right]$ with $v_{0}$ given in (117). The proof that $\left[P_{n}\right]$ implies $\left[P_{n+1}\right]$ with the same $v_{0}, v_{1}, \ldots, v_{2 n}$ as in $\left[P_{n}\right]$ can be carried out in the same way, by the repeated use of Lemma 4.15. Hence we obtain a sequence $\left\{v_{n}\right\}_{n=0}^{\infty}$ of normcontinuous paths $v_{n}:[0,1] \rightarrow \mathcal{U}\left(\mathcal{A}_{\Gamma}^{G}\right)$ with $v_{n}(0)=\mathbb{I}_{\mathcal{A}_{\Gamma}}$ satisfying (120) (121) (122).

We define norm continuous paths $y, z:[0, \infty) \rightarrow \mathcal{U}\left(\mathcal{A}_{\Gamma}^{G}\right)$ by

$$
y(t):=v_{1}(t) v_{3}(t) \cdots v_{2 j-1}(1) v_{2 j+1}(t-[t]), \quad j \leq t<j+1, \quad j=0,1,2, \ldots,
$$

$$
z(t):=v_{0}(t) v_{2}(t) \cdots v_{2 j-2}(1) v_{2 j}(t-[t]), \quad j \leq t<j+1 \quad j=0,1,2, \ldots
$$

Here $[t]$ denotes the largest integer less than or equal to $t$. Then as in section 2 of [KOS, for any $a \in \mathcal{A}_{\mathrm{loc}, \Gamma}$, the limit

$$
\gamma_{0}(a):=\lim _{t \rightarrow \infty} \operatorname{Ad}(z(t))(a), \quad \gamma_{1}(a):=\lim _{t \rightarrow \infty} \operatorname{Ad}(y(t))(a)
$$

exist because of (122) and the fact that $\mathcal{S}_{\Lambda_{n}} \subset \mathcal{F}_{n}$. These limit define endomorphisms $\gamma_{0}, \gamma_{1}$ on $\mathcal{A}_{\Gamma}$. Furthermore, because of (122) and the fact that

$$
\operatorname{Ad}\left(v_{n-1}(1)^{*} v_{n-3}(1)^{*} \cdots\right)\left(\mathcal{S}_{\Lambda_{n}}\right) \subset \mathcal{F}_{n},
$$

by the definition (118) and (119), for any $x \in \mathcal{A}_{\mathrm{loc}, \Gamma}$, the limit

$$
\begin{aligned}
& \lim _{j \rightarrow \infty} \operatorname{Ad}\left(v_{2 j}(1)^{*} v_{2 j-2}(1)^{*} \cdots v_{0}(1)^{*}\right)(x)=: a_{x} \\
& \lim _{j \rightarrow \infty} \operatorname{Ad}\left(v_{2 j-1}(1)^{*} v_{2 j-3}(1)^{*} \cdots v_{1}(1)^{*}\right)(x)=: b_{x}
\end{aligned}
$$

exist. For these limits, we have $\gamma_{0}\left(a_{x}\right)=x$, and $\gamma_{1}\left(b_{x}\right)=x$, for all $x \in \mathcal{A}_{\mathrm{loc}, \Gamma}$. Therefore, $\gamma_{0}$ and $\gamma_{1}$ are automorphisms. By 1., 2. of $\left[P_{n}\right]$, we also have

$$
\varphi_{0} \circ \gamma_{0}=\left.\hat{\varphi}_{\xi_{0}}\right|_{\mathcal{A}_{\Gamma}} \circ \gamma_{0}=\left.\hat{\varphi}_{\xi_{1}}\right|_{\mathcal{A}_{\Gamma}} \circ \gamma_{1}=\varphi_{1} \circ \gamma_{1} .
$$

Let $\Xi_{\Gamma}$ be an automorphism given by $\Xi_{\Gamma}:=\gamma_{0} \circ \gamma_{1}^{-1}$ on $\mathcal{A}$. Define a norm-continuous path $w:[0, \infty) \rightarrow \mathcal{U}\left(\mathcal{A}_{\Gamma}^{G}\right)$ by

$$
w(t):=z(t) y(t)^{*}, \quad t \in[0, \infty) .
$$

We have

$$
\Xi_{\Gamma}(x)=\gamma_{0} \circ \gamma_{1}^{-1}(x)=\lim _{t \rightarrow \infty} \operatorname{Ad}(w(t))(x), \quad x \in \mathcal{A}_{\Gamma},
$$

and $w(0)=\mathbb{I}$. From (131), we have $\varphi_{0} \circ \Xi_{\Gamma}=\varphi_{1}$. This completes the proof.

\section{Proof OF THE MAIN THEOREM}

Now we are ready to prove Theorem 1.11, Let $\omega_{0}$ and $\omega_{1}$ be elements of $S P G(\mathcal{A})$.

Proof of "if" part of Theorem 1.11. Suppose that $c_{\omega_{0}, R}=c_{\omega_{1}, R}$. From Lemma 2.5. we have $c_{\omega_{0}, L}=c_{\omega_{1}, L}$. For each $\zeta=L, R$ and $i=0,1$, let $\left(\mathcal{L}_{\omega_{i}, \varsigma}, \rho_{\omega_{i}, \varsigma}, u_{\omega_{i}, \varsigma}, \sigma_{\omega_{i}, \varsigma}\right)$ be a quadruple associated to $\left(\left.\omega_{i}\right|_{\mathcal{A}_{\varsigma}}, \tau_{\varsigma}\right)$. By Remark 1.8, we may assume that $\sigma_{R}:=\sigma_{\omega_{0}, R}=\sigma_{\omega_{1}, R}$ and $\sigma_{L}:=\sigma_{\omega_{0}, L}=\sigma_{\omega_{1}, L}$. For each $\zeta=L, R$ and $i=0,1$, the triple $\left(\mathcal{L}_{\omega_{i}, \varsigma}, \rho_{\omega_{i}, \varsigma}, u_{\omega_{i}, \varsigma}\right)$ is an irreducible covariant representations of the twisted $C^{*}$-dynamical system $\Sigma_{\Gamma_{\varsigma}}^{\left(\sigma_{\varsigma}\right)}$. By Lemma 1.1, $u_{\omega_{i}, \varsigma}$ has an irreducible decomposition given by a set of Hilbert spaces $\left\{\mathcal{K}_{\gamma, i, \varsigma} \mid \gamma \in \mathcal{P}_{\sigma_{\varsigma}}\right\}$. For each $\varsigma=L, R$, fix some 
$\alpha_{\varsigma} \in \mathcal{P}_{\sigma_{\varsigma}}$. The spaces $\mathcal{K}_{\alpha_{\varsigma}, i, \varsigma} i=0,1$ are non-zero because of Proposition 3.1. Fix unit vectors $\xi_{i, \varsigma} \in \mathcal{K}_{\alpha_{\varsigma}, i, \varsigma}$ for each $\varsigma=L, R$ and $i=0,1$.

For each $\varsigma=L, R$ and $i=0,1$, let $\hat{\varphi}_{\xi_{i, \varsigma}}$ be a state on $\mathcal{B}\left(\mathcal{H}_{\alpha_{\varsigma}}\right) \otimes C^{*}\left(\Sigma_{\varsigma}^{\left(\sigma_{\varsigma}\right)}\right)$ given by $\left(\mathcal{L}_{\omega_{i}, \varsigma}, \rho_{\omega_{i}, \varsigma}, u_{\omega_{i}, \varsigma}, \sigma_{\omega_{i}, \varsigma}\right)$ (defined in Notation 4.1 (152) with $\mathcal{H}, \pi, u, \xi$ replaced by $\left.\mathcal{L}_{\omega_{i}, \varsigma}, \rho_{\omega_{i}, \varsigma}, u_{\omega_{i}, \varsigma}, \xi_{i, \varsigma}\right)$. Let $\varphi_{i, \varsigma}$ be the restriction of $\hat{\varphi}_{\xi_{i, \varsigma}}$ onto $\mathcal{A}_{\Gamma_{\varsigma}}$. By the definition, $\varphi_{0, \varsigma}, \varphi_{1, \varsigma}$ are quasi-equivalent to $\left.\omega_{0}\right|_{\mathcal{A}_{\varsigma}},\left.\omega_{1}\right|_{\mathcal{A}_{\varsigma}}$, respectively.

By Proposition 4.2, there exist $\Xi_{\varsigma} \in \operatorname{AInn}^{G}\left(\mathcal{A}_{\varsigma}\right)$ such that $\varphi_{1, \varsigma}=\varphi_{0, \varsigma} \circ \Xi_{\varsigma}, \varsigma=$ $L, R$. Recall that $\omega_{0}, \omega_{1}$ are quasi-equivalent to $\left.\left.\omega_{0}\right|_{\mathcal{A}_{L}} \otimes \omega_{0}\right|_{\mathcal{A}_{R}}$ and $\left.\left.\omega_{1}\right|_{\mathcal{A}_{L}} \otimes \omega_{1}\right|_{\mathcal{A}_{R}}$ respectively from the split property. (Remark 1.5.) Hence we obtain

$$
\begin{aligned}
\omega_{1} & \left.\left.\sim_{\text {q.e. }} \omega_{1}\right|_{\mathcal{A}_{L}} \otimes \omega_{1}\right|_{\mathcal{A}_{R}} \sim_{\text {q.e. }} \varphi_{1, L} \otimes \varphi_{1, R} \\
& =\left(\varphi_{0, L} \circ \Xi_{L}\right) \otimes\left(\varphi_{0, R} \circ \Xi_{R}\right)=\left(\varphi_{0, L} \otimes \varphi_{0, R}\right) \circ\left(\Xi_{L} \otimes \Xi_{R}\right) \\
& \sim_{\text {q.e. }}\left(\left.\left.\omega_{0}\right|_{\mathcal{A}_{L}} \otimes \omega_{0}\right|_{\mathcal{A}_{R}}\right) \circ\left(\Xi_{L} \otimes \Xi_{R}\right) \sim_{\text {q.e. }} \omega_{0} \circ\left(\Xi_{L} \otimes \Xi_{R}\right) .
\end{aligned}
$$

This completes the proof.

Proof of "only if" part of Theorem 1.11. Suppose that $\omega_{0} \sim_{\text {split }, \tau} \omega_{1}$. Then there exist automorphisms $\Xi_{L} \in \operatorname{AInn}^{G}\left(\mathcal{A}_{L}\right)$ and $\Xi_{R} \in \operatorname{AInn}^{G}\left(\mathcal{A}_{R}\right)$ such that $\omega_{1}$ and $\omega_{0} \circ\left(\Xi_{L} \otimes \Xi_{R}\right)$ are quasi-equivalent. From the split property, we have $\omega_{1} \sim_{\text {q.e. }}$ $\left.\left.\omega_{1}\right|_{\mathcal{A}_{L}} \otimes \omega_{1}\right|_{\mathcal{A}_{R}}$ and $\omega_{0} \circ\left(\Xi_{L} \otimes \Xi_{R}\right) \sim_{\text {q.e. }}\left(\left.\omega_{0}\right|_{\mathcal{A}_{L}} \circ \Xi_{L}\right) \otimes\left(\left.\omega_{0}\right|_{\mathcal{A}_{R}} \circ \Xi_{R}\right)$. Combining these, we see that $\left.\omega_{1}\right|_{\mathcal{A}_{R}}$ and $\left.\omega_{0}\right|_{\mathcal{A}_{R}} \circ \Xi_{R}$ are quasi-equivalent.

For each $\zeta=L, R$ and $i=0,1$, let $\left(\mathcal{L}_{\omega_{i}, \varsigma}, \rho_{\omega_{i}, \varsigma}, u_{\omega_{i}, \varsigma}, \sigma_{\omega_{i}, \varsigma}\right)$ be a quadruple associated to $\left(\left.\omega_{i}\right|_{\mathcal{A}_{\varsigma}}, \tau_{\varsigma}\right)$. From $\left.\left.\omega_{1}\right|_{\mathcal{A}_{R}} \sim_{\text {q.e. }} \omega_{0}\right|_{\mathcal{A}_{R}} \circ \Xi_{R}, \rho_{\omega_{0}, R} \circ \Xi_{R}$ is an irreducible $*-$ representation of $\mathcal{A}_{R}$ on $\mathcal{L}_{\omega_{0}, R}$, which is quasi-equivalent to the GNS representation of $\left.\omega_{1}\right|_{\mathcal{A}_{R}}$. Furthermore, the projective unitary representation $u_{\omega_{0}, R}$ of $G$ with 2cocycle $\sigma_{\omega_{0}, R}$ satisfies

$$
\begin{aligned}
\rho_{\omega_{0}, R} \circ \Xi_{R} \circ \tau_{R}(g)(a) & =\rho_{\omega_{0}, R} \circ \tau_{R}(g) \circ \Xi_{R}(a) \\
& =\operatorname{Ad}\left(u_{\omega_{0}, R}(g)\right) \circ \rho_{\omega_{0}, R} \circ \Xi_{R}(a), \quad a \in \mathcal{A}_{R}, \quad g \in G .
\end{aligned}
$$

From this, $\left(\mathcal{L}_{\omega_{0}, R}, \rho_{\omega_{0}, R} \circ \Xi_{R}, u_{\omega_{0}, R}, \sigma_{\omega_{0}, R}\right)$ is a quadruple associated to $\left(\left.\omega_{1}\right|_{\mathcal{A}_{R}}, \tau_{R}\right)$. Hence we obtain $c_{\omega_{1}, R}=c_{\omega_{0}, R}$. This proves the claim.

\section{Appendix A. Basic notation}

For a Hilbert space $\mathcal{H}, B(\mathcal{H})$ denotes the set of all bounded operators on $\mathcal{H}$. If $V: \mathcal{H}_{1} \rightarrow \mathcal{H}_{2}$ is a linear/anti-linear map from a Hilbert space $\mathcal{H}_{1}$ to another Hilbert space $\mathcal{H}_{2}$, then $\operatorname{Ad}(V): B\left(\mathcal{H}_{1}\right) \rightarrow B\left(\mathcal{H}_{2}\right)$ denotes the map $\operatorname{Ad}(V)(x):=V x V^{*}$, $x \in B\left(\mathcal{H}_{1}\right)$.

For a set $\mathcal{X}, \mathcal{F} \Subset \mathcal{X}$ means that $\mathcal{F}$ is a finite subset of $\mathcal{X}$. For a finite set $S,|S|$ indicates the number of elements in $S$.

For a $C^{*}$-algebra $\mathcal{B}$, we denote by $\mathcal{B}_{1}$ the set of all elements in $\mathcal{B}$ with norm less than or equal to 1 and by $\mathcal{B}_{+, 1}$ the set of all positive elements in $\mathcal{B}_{1}$. For a state $\omega$, $\varphi$ on a $C^{*}$-algebra $\mathcal{B}$, we write $\omega \sim_{\text {q.e. }} \varphi$ when they are quasi-equivalent. We denote by Aut $\mathcal{B}$ the group of automorphisms on a $C^{*}$-algebra $\mathcal{B}$. For a unital $C^{*}$-algebra $\mathcal{B}$, the unit of $\mathcal{B}$ is denoted by $\mathbb{I}_{\mathcal{B}}$. For a Hilbert space we write $\mathbb{I}_{\mathcal{H}}=\mathbb{I}_{\mathcal{B}(\mathcal{H})}$. For a unital $C^{*}$-algebra $\mathcal{B}$, by $\mathcal{U}(\mathcal{B})$, we mean the set of all unitary elements in $\mathcal{B}$. For a Hilbert space we write $\mathcal{U}(\mathcal{H})$ for $\mathcal{U}(\mathcal{B}(\mathcal{H}))$. For a $C^{*}$-algebra $\mathcal{B}$ and $v \in \mathcal{B}$, we set $\operatorname{Ad}(v)(x):=v x v^{*}, x \in \mathcal{B}$. For a state $\varphi$ on $\mathcal{B}$ and a $C^{*}$-subalgebra $\mathcal{C}$ of $\mathcal{B},\left.\varphi\right|_{\mathcal{C}}$ indicates the restriction of $\varphi$ to $\mathcal{C}$. 


\section{Appendix B. FACTS From [KOS] AND [FKK]}

In this section, we list up facts used/proven in [KOS] and [FKK].

Lemma B.1. Let $\varphi_{i}, i=0,1$ be pure states on a simple unital $C^{*}$-algebra $\mathcal{A}$ with GNS triple $\left(\mathcal{H}_{i}, \pi_{i}, \Omega_{i}\right)$. Then for all $\mathcal{F} \Subset \mathcal{A}$ and $\varepsilon>0$ there exists an $f \in \mathcal{A}_{+, 1}$ and a unit vector $\zeta \in \mathcal{H}_{1}$ such that

$$
\pi_{1}(f) \zeta=\zeta, \quad\left\|f\left(a-\varphi_{0}(a) \mathbb{I}_{\mathcal{A}}\right) f\right\|<\varepsilon, \quad \text { for all } \quad a \in \mathcal{F} .
$$

Proof. See proof of Lemma 2.3 of [FKK].

By a basic consideration of 2-dimensional Hilbert space, we obtain the following.

Lemma B.2. For any $\varepsilon>0$, a Hilbert space $\mathcal{H}$, and unit vectors $\xi_{1}, \xi_{2} \in \mathcal{H}$ with $\left\|\xi_{1}-\xi_{2}\right\|<\frac{1}{\sqrt{2}} \varepsilon$, there exists a unitary $V$ on $\mathcal{H}$ such that $\xi_{2}=V \xi_{1}$ and $\left\|V-\mathbb{I}_{\mathcal{H}}\right\|<\varepsilon$.

Notation B.3. For any $\varepsilon>0$, there exists a $\delta_{1, a}(\varepsilon)>0$ satisfying the following: For any $t \in \mathbb{R}$ with $|t| \leq \delta_{1, a}(\varepsilon)$, we have $\left|e^{i t}-1\right|<\varepsilon$. We will fix such $\delta_{1, a}(\varepsilon)>0$ for each $\varepsilon>0$.

Theorem B.4. For any $\varepsilon>0$, there exists a $\delta_{2, a}(\varepsilon)>0$ satisfying the following: For any Hilbert space $\mathcal{H}$, unital $C^{*}$-algebra $\mathcal{A}$ acting irreducibly on $\mathcal{H}$, and $\xi, \eta \in \mathcal{H}$, if there is a unitary operator $v$ on $\mathcal{H}$ satisfying $\|v-\mathbb{I}\|<\delta_{2, a}(\varepsilon)$ and $\eta=v \xi$, there exists a self-adjoint $h \in \mathcal{A}$ such that $e^{i h} \xi=\eta$ and $\|h\| \leq \delta_{1, a}(\varepsilon)$.

Proof. This is a quantitative version of the Kadison transitivity theorem. It can be obtained by precise estimation of approximation in each step of the proof of the Kadison transitivity theorem.

The following Theorem is called Glimm's Lemma

Theorem B.5. Let $\mathcal{A}$ be a unital $C^{*}$-algebra acting on a Hilbert space $\mathcal{H}$. Suppose that the intersection of $\mathcal{A}$ and the set of compact operators on $\mathcal{H}$ is $\{0\}$. Then for any $\varepsilon>0$, a pure state $\varphi$ of $\mathcal{A}$, a finite dimensional subspace $\mathcal{K}$ of $\mathcal{H}$ and a finite subset $\mathcal{F}$ of $\mathcal{A}$, there exists a unit vector $\xi$ in the orthogonal complement of $\mathcal{K}$ such that

$$
|\varphi(x)-\langle\xi, x \xi\rangle|<\varepsilon, \quad \text { for all } \quad x \in \mathcal{F} .
$$

Let us recall the following fact from $[\mathrm{KOS}$.

Lemma B.6. For any $\varepsilon>0$ and $n \in \mathbb{N}$, there exists a $\delta_{3, a}(\varepsilon, n)>0$ satisfying the following.: Let $\mathcal{H}$ be an infinite dimensional Hilbert space and $\mathcal{A}$ a unital $C^{*}$ algebra acting irreducibly on $\mathcal{H}$. Let $\left\{x_{i}\right\}_{i=1}^{n} \subset \mathcal{A}$ be a finite sequence satisfying $\sum_{i=1}^{n} x_{i} x_{i}^{*}=1$. Let $\xi, \eta \in \mathcal{H}$ be unit vectors such that $x_{i}^{*} \xi$ and $x_{j}^{*} \eta$ are orthogonal for any $i, j=1, \ldots, d$ and

$$
\left|\left\langle\xi, x_{i} x_{j}^{*} \xi\right\rangle-\left\langle\eta, x_{i} x_{j}^{*} \eta\right\rangle\right|<\delta_{3, a}(\varepsilon, n), \quad i, j=1, \ldots, n .
$$

Then there exists a positive element $h \in \mathcal{A}_{+, 1}$ such that

$$
\begin{gathered}
\|\bar{h}(\xi+\eta)\|<\frac{1}{4 \sqrt{2}} \delta_{2, a}\left(\frac{\varepsilon}{8}\right) e^{-\pi}, \quad \text { and } \\
\|(\mathbb{I}-\bar{h})(\xi-\eta)\|<\frac{1}{4 \sqrt{2}} \delta_{2, a}\left(\frac{\varepsilon}{8}\right) e^{-\pi}
\end{gathered}
$$


hold for

$$
\bar{h}:=\sum_{j=1}^{n} x_{j} h x_{j}^{*} .
$$

Here the function $\delta_{2, a}$ is given in Theorem B.4.

Proof. See section 3 of [KOS] for the proof.

\section{REFERENCES}

[BC] Erik Bédos and Roberto Conti, On discrete twisted $\mathrm{C}^{*}$-dynamical systems, Hilbert $\mathrm{C}^{*}$ modules and regularity, Münster J. Math. 5 (2012), 183-208. MR3047632

[B] Ola Bratteli, Inductive limits of finite dimensional $C^{*}$-algebras, Trans. Amer. Math. Soc. 171 (1972), 195-234, DOI 10.2307/1996380. MR312282

[BR1] O. Bratteli and D. W. Robinson. Operator Algebras and Quantum Statistical Mechanics 1. Springer-Verlag. (1986).

[BR2] Ola Bratteli and Derek W. Robinson, Operator algebras and quantum statistical mechanics. 2: Equilibrium states. Models in quantum statistical mechanics, 2nd ed., Texts and Monographs in Physics, Springer-Verlag, Berlin, 1997. MR 1441540

[E] George A. Elliott, Some simple $C^{*}$-algebras constructed as crossed products with discrete outer automorphism groups, Publ. Res. Inst. Math. Sci. 16 (1980), no. 1, 299-311, DOI $10.2977 /$ prims/1195187509. MR.574038

[FKK] Hajime Futamura, Nobuhiro Kataoka, and Akitaka Kishimoto, Homogeneity of the pure state space for separable $C^{*}$-algebras, Internat. J. Math. 12 (2001), no. 7, 813-845, DOI 10.1142/S0129167X01001015. MR1850673

[GW] Z.-C. Gu and X.-G. Wen, Tensor-entanglement-filtering renormalization approach and symmetry-protected topological order, Phys. Rev. B, 80, 1551312009.

[KOS] Akitaka Kishimoto, Narutaka Ozawa, and Shôichirô Sakai, Homogeneity of the pure state space of a separable $C^{*}$-algebra, Canad. Math. Bull. 46 (2003), no. 3, 365-372, DOI 10.4153/CMB-2003-038-3. MR 1994863

[M1] Taku Matsui, The split property and the symmetry breaking of the quantum spin chain, Comm. Math. Phys. 218 (2001), no. 2, 393-416, DOI 10.1007/s002200100413. MR.1828987

[M2] Taku Matsui, Boundedness of entanglement entropy and split property of quantum spin chains, Rev. Math. Phys. 25 (2013), no. 9, 1350017, 31, DOI 10.1142/S0129055X13500177. MR.3119173

[O] Yoshiko Ogata, $A \mathbb{Z}_{2}$-index of symmetry protected topological phases with time reversal symmetry for quantum spin chains, Comm. Math. Phys. 374 (2020), no. 2, 705-734, DOI 10.1007/s00220-019-03521-5. MR 4072228

[P] Robert T. Powers, Representations of uniformly hyperfinite algebras and their associated von Neumann rings, Ann. of Math. (2) 86 (1967), 138-171, DOI 10.2307/1970364. MR218905

[S] Barry Simon, Representations of finite and compact groups, Graduate Studies in Mathematics, vol. 10, American Mathematical Society, Providence, RI, 1996. MR.1363490

[T] M. Takesaki, Theory of operator algebras. II, Encyclopaedia of Mathematical Sciences, vol. 125, Springer-Verlag, Berlin, 2003. Operator Algebras and Non-commutative Geometry, 6. MR 1943006

Graduate School of Mathematical Sciences, The University of Tokyo, Komaba, TOKYO, 153-8914, JAPAN 